\title{
LIGHT CONE BEHAVIOR OF PERTURBATION THEORY
}

N. Chritst ${ }^{\dagger}$

Columbia Uhiversity, Now York, N. Y. 10027

B. Hasslocher

University of Illinois, Urbona, Ill. 61801

and

A. Muveller

Columbia University, New York, N. Y. 10027

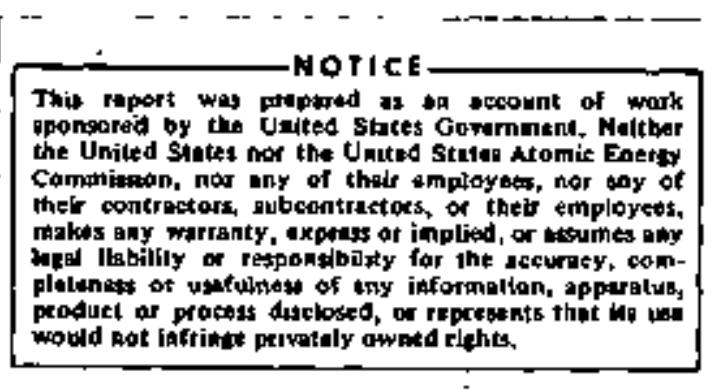

This research was supported in part by the U. 5. Atomic Energy Commission.

† Alfred P. Slaqn Foundation Fellow 


\section{DISCLAIMER}

This report was prepared as an account of work sponsored by an agency of the United States Government. Neither the United States Government nor any agency Thereot, nor any of their employees, makes any warranty, express or implied, or assumes any legal liability or responsibility for the accuracy, completeness, or usefulness of any information, apparatus, product, or process disclosed, or represents that its use would not infringe privately owned rights. Reference herein to any specific commercial product, process, or service by trade name, trademark, manufacturer, or otherwise does not necessarily constitute or imply its endorsement, recommendation, or favoring by the United States Government or any agency thereof. The views and opinions of authors expressed herein do not necessarlly state or reflect those of the United States Government or any agency thereof. 


\section{DISCLAIMER}

Portions of this document may be illegible in electronic image products. Images are produced from the best available original document. 


\begin{abstract}
A techniqui introduced by Symonzik is used to derive a series of equbtions obsyed order by order in perturbation theory by the structure functions $W_{1}$ and $v W_{2}$ entering the eross enction for inelastic electron scottering. These equations relate the $q^{2}, v$ and coupling constent dependence of $W_{1}$ and $v W_{2}$ in o manner reminiscent of the renormalization group results of Gell-Mann and Low. The equations ore used to compute the leading logarithmic contribution to $v W_{2}$ in a theory of fermions coupled to psoudoscolor portictes and a theory of formions coupled to vector particles.
\end{abstract}




\section{INTRODUCTION}

The simple scating behavior ${ }^{\top}$ of the structure functions $W_{1}$ and $v_{2} 2$ observed for $q^{2}$ and mv $\geq 2 \mathrm{Bov}^{2}$ hes caused considerablo interest in the large $q^{2}$ and $v$ depondance of the matrix eloment

$$
\begin{aligned}
& \frac{1}{8=m} \sum_{s= \pm \frac{1}{2}} f \theta^{-i q \cdot x} d^{4} x\left\langle p, s\left|J_{\mu}(x) s_{v}(0)\right| p, s\right\rangle \\
& =\left(\delta_{\mu v}-\frac{q \mu q}{q^{2}}\right) W_{1}\left(q^{2}, \omega\right)+\frac{1}{m^{2}}\left(p^{\mu}-q^{\mu} \frac{p \cdot q}{q^{2}}\right)\left(p^{\nu}-q^{v} \frac{p \cdot q}{q^{2}}\right) w_{2}\left(q^{2}, \omega\right)
\end{aligned}
$$

where $|p, s\rangle$ is a single mucleon stale with four-momentum $p$ and $z$ component of spin s. $J_{\mu}(x)$ is the visol electromagnetic current ${ }^{4}$. In this poper wa investigate the behovior of $W_{1}$, and $v W_{2}$ for large $q^{2}$ and fixed $w=2 m v / q{ }^{2}$ as computed to arbitrary order in the perturbation expansion of a renormalizable field theory.

As is well known 5 , the large $q^{2}$ and $v$ behovior of the matrix elament (1) can be determined from the singularity of the product $J_{\mu}(x) J_{v}(0)$ on the fight cone, $x^{2}=0$. We begin with Wilson's operator exponsion 6,7 for the short distance limit of the product $J_{\mu}\left(\frac{x+y}{2}\right) J_{v}\left(\frac{-x+y}{2}\right)$

$$
\begin{aligned}
& J_{\mu}\left(\frac{x+y}{2}\right) J_{v}\left(\frac{-x+y}{2}\right)=\left(8^{\mu v} \frac{\partial}{\partial x_{a}} \frac{\partial}{\partial x_{a}}-\frac{\partial}{\partial x_{\mu}} \frac{\partial}{\partial x_{v}}\right) \frac{t}{x^{2}+i-x_{0}} \\
& \sum_{n=0}^{N} \sum_{i=0}^{u_{n}} F_{n}^{(i)}\left(x^{2}+i e x_{0}\right) O_{\mu_{1}}^{(i)} \ldots \mu_{n}(y) x_{\mu_{1}} \\
& \left.\cdots x_{\mu_{n}}+R_{N}^{(i)}(x, y)\right\}+\left(8^{\mu \nu} \frac{\partial}{\partial x_{a}} \frac{\partial}{\partial x_{B}}\right.
\end{aligned}
$$




$$
\begin{aligned}
& \left.+\delta_{\alpha \mu} \delta_{\beta v} \frac{\partial}{\partial x_{p}} \frac{\partial}{\partial x_{\rho}}-\frac{\partial}{\partial x_{\alpha}} \frac{\partial}{\partial x_{\mu}} \delta_{\beta v}-\frac{\partial}{\partial x_{\alpha}} \frac{\partial}{\partial x_{v}} \delta_{B \mu}\right)
\end{aligned}
$$

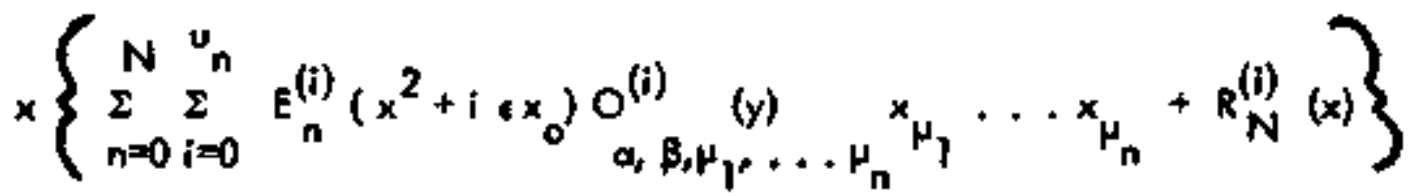

where $o_{a}^{(i)} \ldots a_{n}(y)$ are finite local operotors, traceless and symmetric with respoct to wach poir of Lorentz indices. ${ }^{8} F_{n}^{(i)}\left(x^{2}\right)$ and $E_{n}^{(i)}\left(x^{2}\right)$ are Canumber functions given by a perturbotion expansion of the form

$$
\begin{aligned}
& F_{n}^{(i)}\left(x^{2}\right)=\sum_{l=0}^{\infty} \sum_{r=0}^{l+1} W_{n}^{(i)}(l, r) g^{2 l} \ln ^{j}\left(x^{2}\right) \\
& E_{n}^{(i)}\left(x^{2}\right)=\sum_{l=0}^{\infty} \sum_{r=0}^{l+1} E_{n}^{(i)}(l, r) g^{2 l} \ln ^{r}\left(x^{2}\right)
\end{aligned}
$$

where $g$ is the coupling constont. The remoinder terms $R_{N}^{(i)}(\lambda x, y)$ and $R_{N}^{\prime}(i)(\lambda x, y)$ approach zero os $\lambda^{+N+1}$ for $\lambda$ opproaching zero and $x^{2}=09,10$. The structure functions $W_{1}$ and $v W_{2}$ con be directly determined from the coofftcients $F_{n}^{(i)}\left(x^{2}\right)$, $E_{n}^{(j)}\left(x^{2}\right), 0 \leq n<\infty$, by substituting the expansion (2) into eq. (1) and carrying out the indicated Fourier transformotion.

Using o technique introduced by Symanzik "11, we derive a set of coupled, first order, partial differential equations satisfied by the functions $E_{n}^{(i)}\left(x^{2}\right), 1 \leq i \leq u_{n}$ ond by the functions $F_{n}^{(i)}\left(x^{2}\right), 1 \leq i \leq u_{n}$. The derivation is bosed on the CallonSymanzik ${ }^{12}$ equations obeyed by Green's functions contoining the product $J_{\mu}(x)$ $J_{v}(0)$. The equations obroined are of the sort predicted in other situations by renormolization group 13,14 arguments and connect the $x^{2}$ and coupling constant dapendence of $F_{n}^{(i)}\left(x^{2}\right), E_{n}^{(i)}\left(x^{2}\right)$. The equations don't completely determine the 
functions $E_{n}^{(i)}\left(x^{2}\right), F_{n}^{(i)}\left(x^{2}\right)$ but ane instead constraints which must be obeyod to abitrary order In perturbotion theory. When combined with expltcil calculations in lowest order perturbation theory, the equations directly delermine the coefficients $\Theta_{n}^{(i)}(2, l+1), \mathrm{E}_{n}^{(1)}(l, l+1)$ of the leoding logarithm in $x^{2}$ appearing in every arder of perturbation theory.

These equations abeyed by the coefficients $E_{n}^{(i)}\left(x^{2}\right)$ and $F_{n}^{(j)}\left(x^{2}\right)$ in the Wilson exponsion are derived for two specific field theories in Sect. II. We begin the seiction by roviewing the connection between the light cone behovion of the product $J_{\mu}(x) J_{v}(0)$, spacified by the exponstion (2), and the large $q^{2}$ and $v$ limit of the structure functions $W_{1}$ and $W_{2}$. Then, in Sect. II B, a theory of neutral pteudoesealar particles interacting with charged spin th particles is considered and the equations for the coefficients $E_{n}^{(1)}\left(x^{2}\right)$ and $F_{n}^{(1)}\left(x^{2}\right)$ derived. Next, in Sect. II $C$, the corresponding equations valid for a theory of neutral vector particles interacting with ehargalspin $\frac{1}{2}$ porticles are obtained. In both ceses there are two distinct operators $Q_{\alpha_{1}}^{(t)} \ldots a_{n}, i=1,2$ which oppeor for each $n$ and the resulting equations are two coupled, first onder, portial differential equations. In Sect. III these equations are combined with lowest order perturbotion theory colculations to obtain $\mathrm{E}_{n}^{(1)}\left(\mathrm{x}^{2}\right)$ in the leoding logorithmic approximation for each of these theosies. The revils are identical to those previously ablained from a detailed anolysis of Feynnen amplitudes to all arders in parturbation theory by Gribov and Lipotov. In Sect. IV we discuss the general solution to these equations. First two sets of opproximate equations are considered which ore obeyed by amplitudes in the peseudo-sealar theory containing no solf energy or vertex corrections. One set is volid for all such amplitudes while the other opplies only to those amplitudes which do not contain o two psoudo-sealar intermediate state. Both sets of equations imply a simple power dependence for $E_{n}^{(i)}\left(x^{2}\right)$ 
4

5.

$$
E_{n}^{(1)}\left(x^{2}\right) \sim v_{n}^{1,1}\left(x^{2}\right)^{\nu_{n}^{(1)}}+v_{n}^{1,2}\left(x^{2}\right)^{v_{n}^{(2)}}
$$

for small $x^{2}$ where the power $v_{n}$ is a nontrivial function of $n$ and the $v_{n} i_{i j}$ are constants. Finally the general solution to our equation is obtained for the vector theory, determining the two functions of wo variables $E_{n}^{(1)}\left(x^{2}, g\right), i=1,2$ In terms of seven functions of a single variable. The possibility that there exists a root 8 of the GolfMann Low eigenvalue condition' is investigated and shown to determine somewhat more explicitly the small $x^{2}$ behavior of this solution. 
H. DERIVATION OF EQUATIONS FOR $E_{n}^{(i)}\left(x^{2}\right), F_{n}^{(i)}\left(x^{2}\right)$

In this section we derive a set of first order partiol differential equations obeyed by the funetions $E_{n}^{(i)}\left(x^{2}\right) \quad 1 \leq i \leq u_{n}$ and by the funetions $F_{n}^{(i)}\left(x^{2}\right)$, $1 \leq i \leq v_{n}$, to orbitrary order in perturbation theory. The make up of the operators $O_{a_{1}}^{(1)} \ldots a_{n}$ appoaring in the exponsion (2) and the precise form of the equations to be derived depend, of course, an the particular field theory considered. We will deal explicilly with two distinct theories. Tho first contains a charged spinar field $\psi(x)$ coupled bilinearly to a neutral pseudoscalar field $f(x)$ through the interaction Lograngion $X_{I}(x)=i g(x) Y_{s} f(x) f(x)$. In the sacend theory, the charged spinor fiatd couples to a vactor field $V_{\mu}$ and $Z_{g}(x)=\lg \bar{\psi}(x) \gamma_{p}+(x) V_{\mu}(x)$.

A. RELATION EETWEEN $W_{1}, v W_{2}$ and $E_{n}^{(i)}\left(x^{2}\right), F_{n}^{(i)}\left(x^{2}\right)$

Before deriving these equetions for $E_{n}^{(i)}$ and $F_{n}^{(i)}$ it is waful to rocoll the connection between $W_{1}, v W_{2}$ and the coefficiens $E_{n}^{(i)}$ and $F_{n}^{(i)}$ in the Wilson expansion (2). Consider the invoriant amplitudes $T_{L}$ and $T_{2}$ entering the spin overaged forward Compton seattering amplitude

$$
\begin{aligned}
& \quad \frac{i}{8} \sum_{s= \pm \frac{1}{2}} s^{-i q \cdot x} d^{4} x\langle p, s| T\left(J_{p}(x) J_{v}(0)|p, s\rangle\right. \\
& =-\left(\delta_{p}-\frac{q p q}{q^{2}}\right) T_{L}+\frac{2}{\left(q^{2}\right)^{2}}\left(p^{\mu} p^{v} q^{2}+s^{\mu v}(p \cdot q)^{2}-p^{p} q^{v}(p-q)\right. \\
& \left.=p^{v} q^{p}(p \cdot q)\right) T_{q} .
\end{aligned}
$$

The amplitudes $T_{L}$ and $T_{2}$ are functions of $q^{2}$ and $w$, related to $W_{1}$ and 
7

$\checkmark w_{2}$ by

$$
\begin{aligned}
& w_{1}=\frac{2}{m} i m\left[-T_{L}+2\left(\frac{p \cdot q}{q}\right)^{2} T_{2}\right] \\
& v w_{2}=-\frac{4}{\pi} \operatorname{im}\left[\frac{p \cdot q}{q} T_{2}\right]
\end{aligned}
$$

(6)

(bb)

for $\omega \geq 1$. If the Wilson expansion (2) is wed to evaluate the left hand side of equation (5) and the fourier transform performed, we find that for $q_{p}$ large 16

$$
\begin{aligned}
& T_{2}\left(q^{2}, \omega\right)=\sum_{n=0}^{N}(\omega)^{n} \sum_{i=1}^{u} \tilde{E}_{n}^{(i)}\left(q^{2}\right) c_{n+2}^{(i)}+r_{N}\left(q^{2}, \omega\right) \\
& T_{L}\left(q^{2}, \omega\right)=\sum_{n=0}^{N}(\omega)^{n} \sum_{i=1}^{u_{n}} F(i)\left(q^{2}\right) c_{n}^{(i)}+r_{N}^{\prime}\left(q^{2}, \omega\right)
\end{aligned}
$$

whore

$$
\begin{aligned}
& \tilde{E}_{n}^{(j)}\left(q^{2}\right)=\frac{i}{8}\left(q^{2}\right)^{n+2} \frac{\partial^{n}}{\partial\left(q^{2}\right)^{n}} f d^{4} \times a^{-i q^{-} \times \varepsilon_{n}^{(i)}\left(x^{2}+i c\right)} \\
& \tilde{F}_{n}^{(i)}(q)=\frac{i}{4}(q)^{n+1} \frac{a^{n}}{\partial\left(q^{2}\right)^{n}} \int d^{4} \times \omega^{-i q \cdot x} \times \frac{F_{n}^{(0)}\left(x^{2}+i d\right)}{x^{2}+i c}
\end{aligned}
$$

and

$$
\begin{aligned}
\underset{s= \pm \frac{1}{2}}{\frac{1}{2} \Sigma}\left\langle p_{,} s\right| o_{a_{1}}^{(i)} & \ldots a_{n}|p, s\rangle=c_{n}^{(1)} p_{a_{1}} \ldots p_{a_{n}}(t i)^{n} \\
& +\left(\text { terms containing } \sigma_{q_{1}} a_{j}\right) .
\end{aligned}
$$

For large $q^{2}$ and small o the remainder term ${ }_{N}\left(q^{2}, \omega\right), r_{N}^{\prime}\left(q^{2}, u\right)$ ore of order $\omega^{n+1}$. Note that only the term proportional to

$$
P_{a_{1}} \cdot \cdot P_{n_{n}}
$$
in 
oquation (9) yields leading torms in the Wilson expansion (2) on the light cons. The matrix element (9) of $O_{a_{1}}^{(j)} \ldots a_{n}$ is proportional to the single symmetric traceloss tenser thet san be formed from the four vector $P_{\mu}$. All terms, in this tonsor, olther than the $P_{a_{1}} \ldots a_{n}$ tem contain factors of $p^{2}=-m^{2}$ and therefore give contributions to $T_{L}$ and $T_{2}$ smaller by a factor of $\mathrm{m}^{2} / q^{2} \mathrm{u}^{2}$.

The andytieity of $T_{L}$ and $i_{2}$ in $v$ for fixad $q^{2}$ implien that to any finito order in porturbation theory the limit $N=\infty$ of the sums in equation (7) defines two anolytic function of $\omega$ neor $u=017$

$$
\begin{aligned}
& T_{2}^{A F}\left(q^{2}, u\right)=\sum_{n=0}^{\infty}(\omega)^{n} \sum_{i=0}^{u_{n}} \tilde{E}_{n}^{(i)}\left(q^{2}\right) e_{n+2}^{(i)} \\
& T_{L}^{A F}\left(q^{2}, u\right)=\sum_{n=0}^{\infty}(w)^{n} \sum_{i=0}^{u_{n}} \tilde{F}_{n}^{(i)}\left(q^{2}\right): e_{n}^{(i)} .
\end{aligned}
$$

These asymptotic forms for $T_{L}$ and $T_{2}$ can be continued into the ontire w plone with the exception of branch points of $4= \pm 1$, and used in equation (6) to compule $W_{1}$ and $v W_{2}$ for lame $q^{2}$ and fixed $w>1$. The fomiliar connection botweon the iarge $q^{2}$, fixed $w$ behovior of $T_{L}$ or $T_{2}$ and the $x^{2}=0$ singularity of the coofficionts $E_{n}^{(0)}\left(x^{2}\right), F_{n}^{(i)}\left(x^{2}\right)$ con be wen from equotion (8).

The relationship botween the cooffieients $\tilde{E}_{n}^{(i)}\left(q^{2}\right), \tilde{F}_{n}^{(i)}(q)$ and the asymptotic behovior of the structure functions $W_{1}$ and $v W_{2}$ implied by equotions (6) and (10) con be nootly inverted. Uhing Couchy's theorem eq. (100) can be written

$$
\sum_{j} e_{n+2}^{(1)} \tilde{E}_{n}^{(i)}\left(q^{2}\right)=\frac{i}{2=i} f_{e} d e^{-n-1} \tau_{2}\left(q^{2}+\infty\right)^{A F}
$$

where $c$ is a contour etreling the origin in a counter-elockwise direction. Since $\frac{r_{2}^{A F}}{2}$ 
has branch points in $w$ at $\pm I$ and boven in $w$, wa can opon up the contour to obtain

$$
\sum c_{n+2}^{(0)} \tilde{E}_{n}^{(t)}\left(q^{2}\right)=\frac{2}{-} \int_{i}^{\infty} \omega^{-n-1} d \mathrm{im}_{2}\left(q^{2}, w\right)^{A F}
$$

or using eq. (60)

$$
\begin{aligned}
\sum c_{n+2}^{(i)} \tilde{E}_{n}^{(0)}\left(q^{2}\right) & =\int_{i}^{\infty} w^{-n-2} v W_{2}\left(q^{2}, \omega\right)^{A f} d \psi \\
& =\int_{0}^{1}\left(\frac{1}{\omega}\right)^{n} v W_{2}\left(q^{2}, \omega\right)^{A f} d\left(\frac{1}{\omega}\right),
\end{aligned}
$$

likewise

$$
\sum c_{n}^{(i)} \tilde{F}_{n}^{(i)}\left(q^{2}\right)=f_{i}^{\infty} \omega^{-n-1}\left[\frac{\omega}{2} v w_{2}^{A F}\left(q^{2}, \omega\right)-m w_{1}^{A F}\left(q^{2}, \omega\right)\right] d \omega .
$$

Equation (13) intarpretas the Callon-Groas and Cormwall-Norton aum rule ${ }^{18}$ in the longuage of the Wilson exponsion. It also identifies $\Sigma c_{n+2}^{(i)} \tilde{E}_{n}^{(i)}\left(q^{2}, w\right)$ as the Moltin tronsform of $v W_{2}\left(q^{2}, u\right)^{A F}$ with respect to the variable $1 / \omega$. This transformation can be inverted, giving

$$
v W_{2}\left(q^{2} \cdot \psi^{A F}=\frac{-i}{2 *} \int_{6-i \infty}^{6+i \infty} d n \omega^{n+1}\left[\sum c_{n+2}^{(i)} \tilde{E}_{n}^{(i)}\left(q^{2}\right)\right]\right.
$$

and similorly

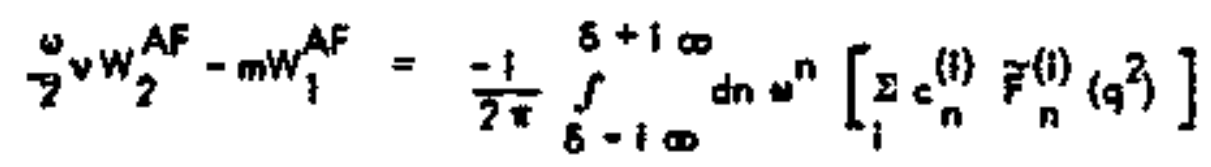

for suffteiently lags, real positive 6 . 


\section{B. PSEUDO-SCALAR THEORY}

Lot us now consider the peoude-sealer caso, specifled by tha Loprongion

$$
\begin{aligned}
X= & -\bar{\phi}\left(\gamma_{\mu} \partial_{\mu}+m\right) \phi-\frac{1}{2} \partial_{\mu} \phi a_{\mu} \phi-\frac{1}{2} \mu^{2} \phi^{2}+i \delta \bar{\phi} \gamma_{s} \phi+\frac{1}{4 !} h \phi^{4} \\
& + \text { (countor torms); }
\end{aligned}
$$

in and $\mu$ ore the physicol mosses of the spin $\frac{1}{2}$ ond the psoudo-sealor porticles while and $h$ are renormolized coupling constants. The renormalization procedure is specified in Appendix A.

The atorting point of our derivation is the Collan-Symenzik equations ${ }^{12}$ for the motrix elemants

$$
\begin{aligned}
& \Gamma_{\mu \nu}^{(1)}(p, x)=\frac{i}{2} f 0^{i} p \cdot(z-y) d^{4} z d^{4} y \cdot(p)_{\delta \sigma}
\end{aligned}
$$

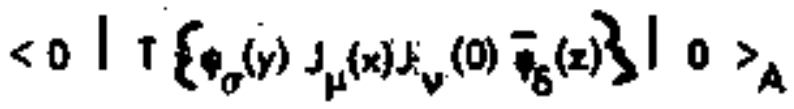

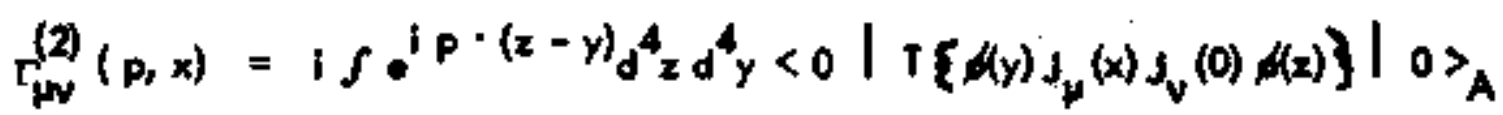

where the subeript A means thot the propogotors corresponding to axternal lines have. been romoved. The Collan-Symanztk equations obeyed by these motrix elements and derived in Appendix A are

$$
D_{1} \Gamma_{\mu v}^{(t)}(x, p)=\Delta \Gamma_{\mu v}^{(i)}(x, p)
$$

for $1=1,2$. The differntlol operotor $D_{1}$ is given by 


$$
D_{i}=m^{2} \frac{\partial}{\partial m^{2}}+\mu^{2} \frac{\partial}{\partial \mu^{2}}+\beta \frac{\partial}{\partial \theta}+\beta^{\prime} \frac{\partial}{\partial h}-2 \gamma_{i}
$$

whilo 19

$$
\begin{aligned}
& \Delta I_{N V}^{(1)}(x, p)=\frac{1}{2} f e^{i p \cdot(z-\gamma)} d^{4} z d^{4} \gamma(p)_{\delta \sigma} \\
& \left.<0\left|T\left\{\varphi_{o}(y) J_{\mu}(x) J_{v}(0) \cup \bar{\psi}_{\delta}(x)\right\}\right| 0\right\rangle_{A} \\
& \left.\Delta F_{\mu v}^{(2)}(x, p)=f e^{i \cdot p(z-y)} d^{4} z d^{4} y<0\left|\tau\left\{d(y)_{\mu}(x) \alpha_{V}(0) \cup \phi(z)\right\}\right| 0\right\rangle_{A}
\end{aligned}
$$

The operator $u$, in the notation of Zimmermon ${ }^{20}$, is

$$
u=\frac{1}{2} \int d^{4} x\left\{m \delta_{1} N[\psi(x) \phi(x)]+\mu^{2} \delta_{2} N[f(x) d(x)]\right\}
$$

where the symbol $N$ indicates the inclusion of subtraction terms, choosen in a.manner specified in Appondix A, so that all matrix eloments of $u$ are finite. The dimensiontess constons $\beta, \beta^{\prime}, \gamma_{1}, \gamma_{2}, \delta_{1}, \delta_{2}$ are functions of $g, h$, and $m / \mu$ and can be computed to arbitrary order in perturbation theary. The Callan-Symonzik equotions (1) are exact bul not very useful as they stand since they relote the behavior of the quantities of interest, (16), to that of two now unknown functions (19). Howevar, if we consider the small $x_{\mu}$ limit of equation (17) and substitute the Witson expomiton (2) into both the right and loft hend sides, then we find that the smoll $x_{j p}$ dependence of both sides is determined by the some funetlons $E_{n}^{(i)}\left(x^{2}\right)$ and $F_{n}^{(I)}\left(x^{2}\right) 11 ; 21$.

The resulting equations ore 
12

$$
D_{i}\left\{\sum_{n=1 j=1}^{\infty} \sum_{2 n}^{u_{2 n}}(j) 2^{\left(x^{2}\right)} b_{2 n}^{i, j}(x \cdot p)^{2 n-2}\right\}=\sum_{n=1}^{\infty} \sum_{j=1}^{u_{2 n}} E_{2 n-2}^{(j)}\left(x^{2)} q_{2 n}^{j, j}(x \cdot p)^{2 n-2}\right.
$$

(21)

for $1=1,2$ and $p^{2}=0$. The constants $o_{n}, j$ and $b_{n}^{i} j$ are related to the relevant matrix elements of ${ }^{(t)}$

$$
\begin{aligned}
& a_{1} \ldots a_{n} \text { by }
\end{aligned}
$$

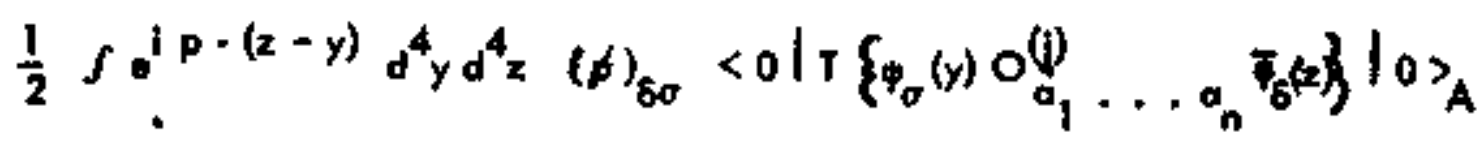

$$
\begin{aligned}
& =b_{n}^{1, j} p_{a_{1}} \ldots p_{a_{n}}(t)^{n}+\text { (terms containing } b_{a_{j} a_{j}} \text { ) } \\
& \left.f e^{i p \cdot(z+y)} d^{4} y d^{4} z<0\left|\tau\left\{(y) O a_{1}^{(j)} \ldots a_{n} \quad \alpha(z)\right\}\right| 0\right\rangle_{A}
\end{aligned}
$$

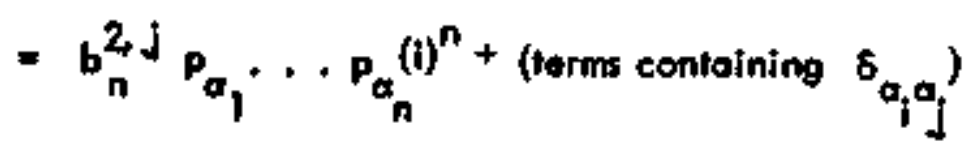

$$
\begin{aligned}
& \left.\frac{1}{2} f e^{i p-y)} d^{4} y d^{4} z(x)_{\delta \sigma}<0 \mid T f_{\sigma}(y) O a_{1} \ldots q_{n} u \phi_{\delta}(z)\right\}|0\rangle_{A} \\
& =a_{n}^{1, j} p_{a_{1}} \cdots p_{a_{n}}(t)^{n}+\text { (teas containing } \delta_{a_{i} a_{j}} \text { ) } \\
& f e^{i p \cdot(z-y)} d^{4} y d^{4} z<0\left|\tau\left\{d(y) o_{a_{1}}^{(j) \ldots \alpha_{n}} u d(z)\right\}\right| 0>_{A} \\
& =a_{n}^{2,} P_{a_{1}} \cdots P_{a_{n}}(i)^{n}+\left(\text { terms containing } \delta_{a_{i j}}\right)
\end{aligned}
$$

for $p^{2}=0$ and $1 \leq a_{i} \leq 3,1 \leq i \leq n$ and $n$ oven. Bose symmetry and charge conjugation invariance imply that the left hand sides of these equations yenish for odd $n$. 
In equation (22) we use $p^{2}=0$ so thet the quantities $a_{n}^{i, j}$ and $b_{n}^{i, d}$ depend only on $\mathrm{m}^{2} / \mu^{2}, g, h$. Equoting the coefficionts of equal powers of $(x-p)^{n}$, we obtoin a series of equations diagonal in the index $n$

$$
D_{i}\left\{\begin{array}{l}
\Sigma E_{n}^{(j)}-2 \\
j
\end{array} x_{n}^{2} b_{n}^{i, j} \xi=\sum_{j} E_{n-2}^{(j)} a_{n}^{i, j}\right.
$$

$2 \leq n \leq \infty, i=1, \ldots, u_{n}$, for even $n$. Equations (21) and (23) are also obeyed by $F_{n}^{(i)}\left(x^{2}\right)$ and $0 \leq n \leq \infty$. These equotions can be fourier tronsformed yielding identical equations for the quantilies $\tilde{E}_{n}^{(i)}\left(q^{2}\right)$

$$
D_{i}\left\{\sum_{j} \tilde{E}_{n-2}^{(j)}\left(q^{2}\right) b_{n}^{i, j}\right\}=\Sigma \tilde{E}_{n-2}^{(j)}\left(q^{2}\right) a_{n}^{i, j},
$$

which ore also obeyed by the functions $F_{n}^{(i)}\left(q^{2}\right)$.

Lat us now determin explicitly the operators which appear in the Wilson exponsion (2) for the particular theory at hond. Because of the requirements of symmetry in the Lorentz indices and the absence of $b_{a_{1}} a_{j}$ foctors, there are only two $\mathrm{N}^{\text {th }}$ rank tensor operators with the smallest dimension which ean be formed 22

$$
\begin{aligned}
& ,(1) \ldots a_{n}(y)=-\frac{1}{4 n}\left(1+(-1)^{n}\right) \underset{j=1}{x} N\left[\Phi(y), a_{a_{1}} \cdots a_{a_{j}}, 1 \gamma_{a_{j}} a_{a_{j}+1} \cdots a_{a_{n}} \phi(y)\right] \\
& + \text { (terms containing } \delta_{0, a_{1}} \text { ) } \\
& o_{a_{1}}^{(2)} \ldots \alpha_{n}(y)=\frac{1}{2} N\left[f(y) a_{a_{1}} \ldots a_{a_{n}} \not(y)\right]+\left(\text { torms containing } \delta_{a_{i} a_{j}}\right)
\end{aligned}
$$


where the symbel $\mathbf{N}$ again indicates that sufficient subtractions hove been mode so thet the resulting operator is finite. The subtroctions will be chosen so that

$$
b_{n}^{i, j}\left(g, h, m^{2} / p^{2}\right)=\delta_{i j} \text {. }
$$

The equations oboyed by $\tilde{E}_{n}^{(t)}(q)$ for $i=1,2,2 \leq n \leq \infty$ (and $\tilde{F}_{n}^{(i)}\left(q^{2}\right), t=1,2$, $0 \leq n \leq \infty)$ then become

$$
D_{i} \tilde{E}_{n-2}^{(i)}\left(q^{2}\right)=\sum_{j=1,2} q_{n}^{i, j} \tilde{E}_{n-2}^{(i)}\left(q^{2}\right) .
$$

These two coupled first order differential equations can be written as uneoupled second order oquations

$$
\begin{aligned}
& \left(D_{2}-a_{n}^{2,2}\right) \frac{1}{a_{n}^{1,2}}\left(D_{1}-a_{n}^{1,1}\right) \tilde{E}_{n}^{(1)}\left(q^{2}\right)=a_{n}^{2,1} \tilde{E}_{n}^{(1)}\left(q^{2}\right) \\
& \left(D_{1}-a_{n}^{1,1}\right) \frac{1}{a_{n}^{2,1}}\left(D_{2}-a_{n}^{2,2}\right) \vec{E}_{n}^{(2)}\left(q^{2}\right)=a_{n}^{1,2} \tilde{E}_{n}^{(2)}\left(q^{2}\right) .
\end{aligned}
$$

These equations (27 or 28) are the desired equations for the pseudo-scalor theory. They are the generolization of Symonzik's exceptional momentum equation to oll the operators in the Wilson exponsion on the light cone. These squalions will be ned in Section Ifl to compute the leading logarithmic contribution to $\mathrm{vW}_{2}$ and in Soction $N$ to speculate about the exact asymptotic behavior of $\mathrm{W}_{1}$ and $\mathrm{VW} \mathrm{W}_{2}$.

\section{MASSIVE VECTOR THEORY}

We now corsider the theory of a vector field $V_{\mu}$ of moss $\mu$ interaeting with a spln $\frac{1}{2}$ field of moss $m$, specified by the Lograngian 


$$
\begin{aligned}
X(x)= & -\bar{\phi}\left(\gamma_{\mu} a_{\mu}+m\right)_{\varphi}-\frac{1}{4} G_{\mu \nu} G_{\mu \nu}+\operatorname{tg} v_{\mu} \bar{\phi} \gamma_{\mu}-\frac{1}{2} \mu^{2} v_{\mu} v_{\mu} \\
& + \text { (counter terms) }
\end{aligned}
$$

where $g$ is the renormolized coupling constent and

$$
G_{\mu v}(x)+\partial_{\mu} V_{v}(x)-\partial_{v} V_{p}(x)
$$

In analogy with oquation (16) we define the amplinudes

$$
\begin{aligned}
& f_{\mu \nu}^{(1)}(p, x)=\frac{1}{2} \int g^{1} p^{-(z-y)} d^{4} z d^{4} y(f)_{6 a} \\
& \left\langle 0\left|T\left[\psi_{\sigma}(y) J_{\mu}(x) J_{\nu}(0) \bar{\psi}_{\delta}(x)\right\}\right| 0\right\rangle_{A}
\end{aligned}
$$

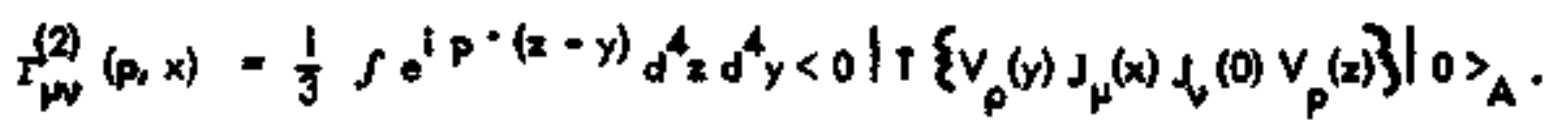

As is thown In Appendix A, these ampitiudes oboy the Collon-Symanzik equotion

$$
D_{i} \Gamma_{\mu v}^{(i)}(p, x)=\Delta r_{\mu v}^{(i)}(p, x)
$$

for $1=1,2$; whore

$$
D_{i}=\left[m^{2} \frac{\partial}{\partial m^{2}}+\mu^{2} \frac{\partial}{\partial \mu^{2}}+\beta \frac{\partial}{\partial g}-4 \gamma_{2}-2 \gamma_{i}\right] \gamma_{3}^{2}
$$

and

$$
\begin{aligned}
& \Delta r_{p N}^{(1)}(x, p)=\frac{1}{2} \int e^{l p \cdot(z-y)} d^{4} z d^{4} y,(p) p \alpha r_{3}^{2} \\
& \left\langle 0\left|+\left\{\phi_{\sigma}(y) \downarrow_{\mu}(x) J_{v}(0) \cup \bar{\phi}_{p}(x)\right\}\right| 0\right\rangle_{A}
\end{aligned}
$$




$$
\begin{aligned}
\Delta r_{\mu v}^{(2)}(x, p)= & \frac{1}{3} \int *^{1 p \cdot(z-y)} d^{4} z d^{4} \gamma \gamma_{3}^{2} \\
& \left.<0\left|+\left\{v_{p}(y) \perp_{\mu}(x) J_{v}(0) \cup v_{p}(z)\right\}\right| 0\right\rangle_{A}
\end{aligned}
$$

for $\beta=g y_{2}$ and

$$
\left.u^{\prime}=\frac{1}{2} \int d^{4} x \ln _{1} \delta_{1} N[\bar{V}(x) \phi(x)]+, \mu^{2} \varepsilon_{2} N\left[v_{p}(x) v_{p}(x)\right]\right) .
$$

Sulustituting the Wilson exponstion (2) into eq. (32) and equating equal powen of $x \cdot p$ we obtoin on oquation identical in form to $* 9 .(23)$

$$
D_{i}\left\{\sum_{j=1}^{u_{n}} E_{n-2}^{(j)} b_{n}^{t, i}\right\}=\sum_{j=1}^{u_{n}} E_{n-2}^{(j)} a_{n}^{j, j}
$$

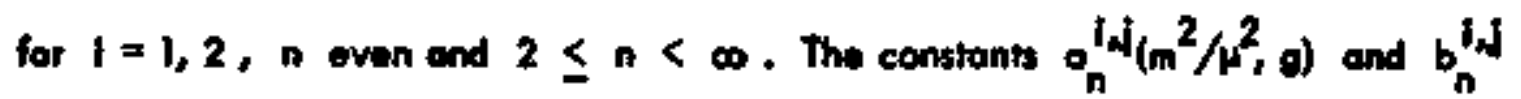
$\left(\mathrm{m}^{2} / \mu^{2}, g\right)$ ore defined by oquations abtained from eq. (22) by roplocing $\mu(y)(z)$ by $1 / 3 V_{p}(y) V_{p}(z)$ and multiplying the laft hond sides of eq. (22e and d) by $r_{3}^{2}$. The longtudinal coofficients $F_{n}^{(i)}$ also oboy eq. (30) for $0 \leq n<\infty$.

Juat as in the pauda-sealar case thore are two types of pperaters then can contribute:

$$
\begin{aligned}
& \partial_{a_{1}}^{(1)} \cdots a_{n}-\frac{1}{s_{n}}\left(i+(-i)^{n}\right) \sum_{j j=1}^{n} N\left[\Phi\left(a_{a_{1}}-i g v_{a_{1}}\right) \ldots \gamma_{a_{j}} \ldots\left(a_{a_{n}}-i g v_{a_{n}}\right)+\right] \\
& + \text { (term contoining } \delta_{\alpha_{1} a_{i}} \text { ) }
\end{aligned}
$$

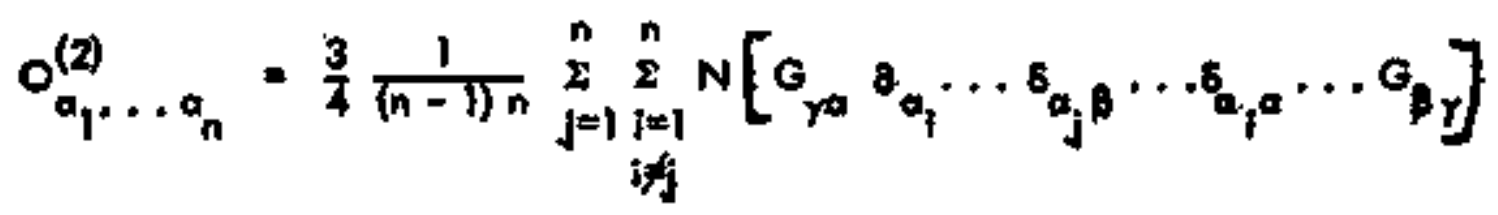

$$
\begin{aligned}
& + \text { (torms containing } 6_{a_{1} a_{j}} \text { ). }
\end{aligned}
$$


The number of possible operators is limited to anly two, for a given $n$, by gave invariance. Both the operator $J_{p}(x) f_{v}(0)$ and the finst three terme of our Lagrangion (29) are inverient under the transformation

$$
\begin{aligned}
& v_{\mu}(x) \rightarrow v_{\mu}(x)+i g a_{\mu} A(x) \\
& \psi(x) \rightarrow e^{i g A(x)} \psi(x) .
\end{aligned}
$$

Although the mess term $-\frac{1}{2} \mu^{2} V_{p} V_{p}$ breaks this gauge symmetry, the leating term in Wilson's expansion (2) are independent of $\mu^{2}$ and hence are left unchanged by the tronsformation (39).

Thus only two saries of functions $E_{n}^{(1)}\left(x^{2}\right)$ and $E_{n}^{(2)}(q)$ are neaded to deturmino $\checkmark W_{2}$ in the lorge $q^{2}$ and $v$ region. If we choose the subtractions requited to make the opentors (37) finite in such a way thot

$$
b_{n}^{i, j}=b_{i j}
$$

and transtorm to monentum space, then eq. (36) bacomes

$$
\left.D_{1} E_{n-2}^{(i)}\left(q^{2}\right)=\underset{j=1,2}{\Sigma} a_{n}^{i, j} Z_{n-2}^{(j)} q^{2}\right)
$$

for $1=1,2,2 \leq n<\infty$, an equation identical in form to that found for the peeudo-scolar theory. This equotion is also aboyed by the functions $f_{n}^{(i)}(q), i=1,2$, $0 \leq n<\infty$. 


\section{PERTURBATION THEORY CALCULATIONS}

In this section wo use the equations derived in Section II to calculate the inelestic electroproduction structure funtion $v W_{2}$ in o leading logarithmic approximation. Vorious authors $15,24,25,26$ have performed such colculations by applying infinite-monentum methods directly to specific classes of Feynman graphs. Such opprocechen require consideroble expertise in the art of extrocting esymptotic behovior from foynman omplitudes. Wo will show how these leading logarishmic resul ts emerge rather triviolly from eq. (27) and (40). Altogether three specific exomplos will be considered: (A) the ladder grophs In the pseuda-scalor theory caleulated by Chang and Fishbers 24 , (B) the complete leading logarithmic behavior in the pueudo-scalar theory, first compulad by Gribov and Lipotor ${ }^{15}$ and (C) the complete loading logarithmic behovior in the vector theory, olso computed by Gribov and Lipotov. 15 .

\section{A. CHANG -fISHaANE CALCULATION}

Chang and Fishbone consider the ladder graphs of Fig. (1) in the looding loogrithmic approximation. In ow notation this meons that they keep oll terms in $\mathrm{E}_{n}^{(1)}\left(q^{2}\right)$ of the form $\left(g^{2}\right)^{r}\left(g^{2} \text { in } q^{2}\right)^{l}$ with $r=0$. Since no intermediate state contoining only

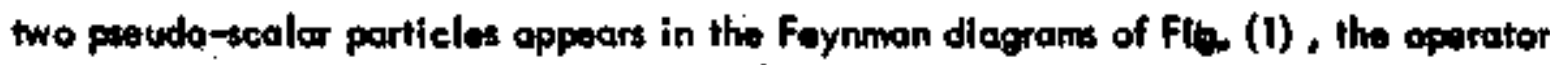

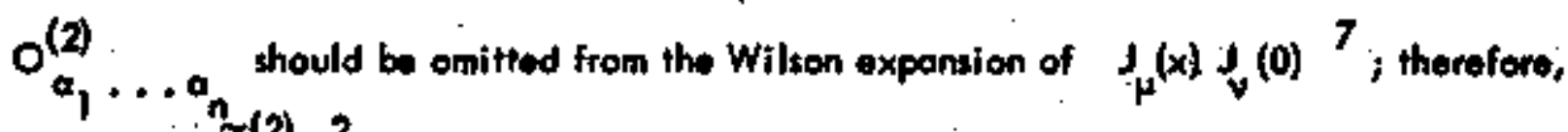
we sat $:{ }_{n}^{n}(2)\left(q^{2}\right)=0$. Furthermore, there ore no propogator or vertex corrections included in this set of grophs so $=g^{\prime}=\gamma_{1}=\gamma_{2}=0$. (In Chang and Fistibene's language we are taking only their outer ralnbew grophs.) Thus eq. (27) becomes simply

$$
\left[m^{2} \frac{\partial}{\partial m^{2}}+\mu^{2} \frac{\partial}{\partial \mu^{2}}\right] \tilde{E}_{n}^{(j)}\left(q^{2}\right)=q_{n+2}^{l, l} E_{n}^{(1)}\left(q^{2}\right) .
$$


Since $\bar{E}_{n}^{i}\left(q^{2}\right)$ is a dimensionless function of $q^{2}, m^{2}$ and $\nu^{2}$ we may reploce

$$
\begin{gathered}
m^{2} \frac{\partial}{\partial m^{2}}+\mu^{2} \frac{\partial}{\partial \mu^{2}} \text { by }+q^{2} \frac{\partial}{\partial q^{2}} \text { to that } 4 q \text {. (41) con be rewritten } \\
q^{2} \frac{\partial}{\partial q^{2}} E_{n}^{(1)}\left(q^{2}\right)=-q_{n+2}^{1,1} E_{n}^{(1)}\left(q^{2}\right)
\end{gathered}
$$

whose solution is

$$
\tilde{E}_{n}^{(1)}\left(q^{2}\right)=v_{n} \exp \left[-a_{n+2}^{1,1} \ln \left(q^{2}\right)\right]=v_{n}\left(q^{2}\right)^{-a_{n+2}^{1,1}} .
$$

To abtain the leoding logarithmic behovior of $\widetilde{E}_{n}^{(1)}\left(q^{2}\right)$ we need only compute the parameter $a_{n+2}^{1,1}$ from oq. (220) to lowest order in $g$ and determine the integrotion constant $v_{n}$ from the $g^{2}=0$ Born termx. This ealeulation of the quentities $0_{n}^{1,1}$ i nvolves the evaluation of a simple lowest order vertex correction and is carried out in Appondix $\mathrm{B}$, yiolding

$$
a_{n+2}^{1, i}=-\frac{g^{2}}{16 \pi^{2}} \frac{1}{(n+2)(n+3)}
$$

for oven $n$. Sines to lowest order in $g$, the $e_{n}^{(j)}$ of eq. (9) equals 1 and

$$
v w_{2}=6\left(1-\frac{1}{4}\right)
$$

eq. (130) implies $v_{n}=1$ so that in looding logarthmic appraximatton

$$
\left.\int_{0}^{1} d\left(\frac{1}{w}\right)\left(\frac{1}{w}\right)^{n+1} v w_{2}^{A F}\left(q^{2}, w\right)=\tilde{E}_{n}^{(1)}(q)=(q)^{2}\right)^{\frac{g^{2}}{16 t^{2}} \frac{1}{(n+2)(n+3)}}
$$

This is exactly the resuit of Cheng and Fishbane for the sel of outer roinbow amplitudes. Thus the Mallin tronsform wed so judiciously by Chang and Fishbone and by Gribor and 
Lipolov is nothing other than the index-continued Wilson expansion, the continuation being andiogous to the Sommerfeld-Watson continuation of a partial wave expansion.

\section{B. GRJBOV -LIPATOV CALCULATION FOR THE PSEUDO-SCALAR THEORY}

We will now find all the leading logarithmic terms in $E_{n}^{(1)^{2}}(q)$ for the $Y_{s}$ thery. The basic equotions for this colculation are given by $\$ q,(27)$ which wo writs in full os

$$
\begin{aligned}
& {\left[-q^{2} \frac{\partial}{\partial q^{2}}+\beta \frac{\partial}{\partial g}+\beta^{\prime} \frac{\partial}{\partial h}-2 \gamma_{1}\right] \tilde{E}_{n}^{(1)}\left(q^{2}\right)=q_{n+2}^{1,1} \tilde{E}_{n}^{(1)}\left(q^{2}\right)+a_{n+2}^{1,2} \tilde{E}_{n}^{(2)}\left(q^{2}\right)} \\
& {\left[-q^{2} \frac{\partial}{\partial q^{2}}+\beta \frac{\partial}{\partial g}+\beta^{\prime} \frac{\partial}{\partial h} \gamma_{2}\right] \tilde{E}_{n}^{(2)}\left(q^{2}\right)=q_{n+2}^{2,1} \tilde{E}_{n}^{(1)}\left(q^{2}\right)+\sigma_{n+2}^{2,2} \tilde{E}_{n}^{(2)}\left(q^{2}\right)}
\end{aligned}
$$

for even $n \geq 0$. Following Gribov and Lipotov we set $h$ ond thenefore $\beta^{\prime}$ equal to zero. (In a regularized theory with no $f^{4}$ interaction term $h$ is of order ${ }^{4}$. ) The quontities $\beta, \gamma_{1}$, and $\gamma_{2}$ can be computed to lowest order in $g$ from oq. (A9) of Appendix A while in Appond $x \mathrm{C}$ the $a_{n}^{i, j}$ are-determined and theit connection with variaus grophs indicated. The results ore

$$
\begin{aligned}
& \beta=\frac{5 g^{3}}{32 m^{2}} \quad \gamma_{1}=\frac{g^{2}}{64 \pi^{2}}, \gamma_{2}=\frac{g^{2}}{16 m^{2}} \\
& a_{n+2}^{1,1}=-\frac{g^{2}}{16 \pi^{2}} \frac{1}{(n+2)(n+3)} ; a_{n+2}^{1,2}=-\frac{g^{2}}{16 t^{2}} \frac{1}{(n+3)} \\
& a_{n+2}^{2,1}=-\frac{g^{2}}{a^{2}} \frac{1}{(n+2)} ; a_{n+2}^{2,2} * 0 .
\end{aligned}
$$

Since in leoding logorithmic approximotion $\tilde{E}_{n}^{(i)}\left(q^{2}\right)$ depends only on $g^{2} \ln \left(q^{2}\right)$ it is 
conventent to introduce the varioble

$$
\xi=-\frac{1}{5} \ln \left[1-\frac{5 q^{2}}{16 \pi^{2}} \ln \left(q^{2}\right)\right] .
$$

The reader will note that

$$
-q^{2} \frac{\partial}{\partial q^{2}}+\beta \frac{\partial}{\partial q}=-\frac{q^{2}}{16 q^{2}} \frac{\partial}{\partial \xi}
$$

when acting on of funetion of $\xi$ alone. Using $4 \%$. (48) and (50), we ean rowrito 4q. (47) क

$$
\begin{aligned}
& \left(\frac{\partial}{\partial \xi}+\frac{1}{2}\right) \tilde{E}_{n}^{(1)}(\xi)=\frac{1}{(n+2)(n+3)} \tilde{E}_{n}^{(1)}(\xi)+\frac{1}{(n+3)} \tilde{E}_{n}^{(2)} \\
& \left(\frac{\partial}{\partial \xi}+2\right) \tilde{E}_{n}^{(2)}(\xi)=\frac{4}{(n+2)} \tilde{E}_{n}^{(0)}(\xi) .
\end{aligned}
$$

which ore equivolent to

$$
\left\{\frac{\partial^{2}}{\partial \xi^{2}}+\left[\frac{5}{2}-\frac{1}{(n+2)(n+3)}\right] \frac{\partial}{\partial \xi}+1-\frac{\sigma}{(n+2)(n+3)}\right) \tilde{E}_{n}^{(1)}(\theta)=0
$$

and $\tilde{E}_{n}^{(2)}(\xi)=(n+3)\left[\frac{a}{\partial \xi}+\frac{1}{2}-\frac{1}{(n+2)(n+3)}\right] \tilde{E}_{n}^{(1)}(k)$.

Eq. (52) implies that $\tilde{\mathbf{E}}_{\boldsymbol{n}}^{(1)}(\xi)$ has the form 


$$
E_{n}^{(1)}(\xi)=C_{n} v^{v_{n}^{*}}+C_{n}^{\prime} e^{v_{n}^{\prime} \xi}
$$

whore

$$
\begin{aligned}
& v_{n}=-\frac{5}{4}+\frac{1}{2(n+2)(n+3)}+\left[\left(\frac{3}{4}+\frac{1}{2(n+2)(n+3)}\right)^{2}+\frac{4}{(n+2)(n+3)}\right]^{\frac{1}{2}} \\
& v_{n}^{\prime}=-\frac{5}{4}+\frac{1}{2(n+2)(n+3)}-\left[\left(\frac{3}{4}+\frac{1}{2(n+2)(n+3)^{2}}+\frac{4}{(n+2)(n+3)}\right]^{\frac{1}{2}} .\right.
\end{aligned}
$$

The integration constants $C_{n}$ and $C_{n}^{\prime}$ are delermined from the known $g^{2}=0$ limit given by the Born terns

$$
\begin{aligned}
& \left.\tilde{E}_{n}^{(1)}(\xi)\right|_{\xi=0}=1 \\
& \left.\tilde{E}_{n}^{(2)}(\xi)\right|_{\xi=0}=0
\end{aligned}
$$

which requires

$$
\begin{aligned}
& c_{n}=\frac{1}{v_{n}^{\prime}-v_{n}^{\prime}}\left[\frac{1}{(n+2)(n+3)}-\frac{1}{2}-v_{n}^{\prime}\right] \\
& C_{n}^{\prime}=\frac{1}{v_{n}^{\prime}-v_{n}}\left[\frac{1}{(n+2)(n+3)}-\frac{1}{2}-v_{n}^{\prime}\right] .
\end{aligned}
$$

A continuation of eq. (54) to complex values of the index $n$, when substituted in Qq. (14a) yields

$$
v W_{2}\left(q^{2}, \omega\right)=\frac{-i}{2 \pi} \int_{\delta-1 \infty}^{5+i \infty} d n w^{n+1}\left[c_{n} e^{v n^{5}}+c_{n}^{2} e^{v} n^{\frac{5}{5}}\right]
$$


in exact agreement with Gribov and Lipatov for the leoding logorithmic behovior of the structure function $\mathrm{VW}$, for deep inelostic scottering of electrons off the field. The reader is referred to the work of Griboy and Lipatov for a discussion of the physical significance, if ony, of this result.

\section{GRIBOV-LIPATOV CALCULATION FOR THE MASSIVE VECTOR THEORY}

Finally wo turn to the ealculation of the looding logorithms in $\mathrm{vW}_{2}$ for the vector theory studied in Sect. II. C. Since this colculation proceeds much as in the pheudo-scolor cose, wo will simply outline the procedure for obtoining the result identical to that of Gribov and Lipatov. The quontitios $=2 \gamma_{2}, \gamma_{1}$ and $\gamma_{3}, \cdots$ determiniod in; Appendix A, con be computed in the faynman gouge to lowest order in with the result

$$
\beta=\frac{g^{3}}{24 \pi^{2}}, \quad \gamma_{1}=\frac{g^{2}}{32 \pi^{2}}, \quad \gamma_{3}=1 \text {. }
$$

Similarly, the constants $a_{n}^{j, j}$ are ovoluated in Appendix $\mathrm{C}$ to order $\mathrm{g}^{2}$ :

$$
\begin{aligned}
& a_{n+2}^{1,1}=-\frac{g^{2}}{8 n^{2}}\left[\frac{1}{(n+2)(n+3)}-\sum_{t=0}^{n} \frac{2}{l+2}\right] \\
& a_{n+2}^{1,2}=\frac{3 g^{2}}{16 n^{2}} \frac{n^{2}+5 n+8}{(n+1)(n+2)(n+3)} \\
& a_{n+2}^{2,1}=\frac{g^{2}}{6 \pi^{2}}\left(\frac{n^{2}+5 n+8}{(n+2)(n+3)(n+4)}\right. \\
& a_{n+2}^{2,2}=0 .
\end{aligned}
$$


Introducing the veriabile

$$
t=-\frac{3}{4} \ln \left[1-\frac{q^{2}}{12 t^{2}} \ln \left(q^{2}\right)\right] \text {. }
$$

we can rowrite oq. (40) for $\breve{E}_{n}^{(i)}\left(q^{2}\right)$ os

$$
\begin{aligned}
& \int \frac{\partial^{2}}{\partial \xi^{2}}-\left[3+\phi_{1}(n+2)+\phi_{3}(n+2)\right] \frac{\partial}{\partial \xi}+\frac{20}{9}+\frac{4}{3}\left[\phi_{1}(n+1)+\psi_{3}(n+2)\right] \\
& \left.-\psi_{2}^{(n+2)}\right\} E_{n}^{(1)}(\xi)=0 \\
& E_{n}^{(2)}=-\frac{g^{2}}{16 \pi^{2}} \frac{1}{a_{n+2}^{1,2}}\left[\frac{\partial}{\partial \xi}-\frac{5}{3}-\phi_{1}^{(n+2)}-\phi_{3}^{(n+2)}\right] \mathrm{E}_{n}^{(1)}
\end{aligned}
$$

where

$$
\begin{aligned}
& \varphi_{1}(i)=\frac{2}{j(j+i)} \\
& \varphi_{2}(i)=\frac{8\left(j^{2}+j+2\right)^{2}}{(j-1) j^{2}(j+1)^{2}(j+2)} \\
& \phi_{3}(i)=-4 \sum_{l=2}^{j} \frac{1}{l}
\end{aligned}
$$

in the notation of Gribov ond Lipatov. These equations, when coupled with the requirement ${ }^{27}$ (56) con be explicitly solved as in the precueding section yielding the Gribóv-lipator resilt. 


\section{GENERAL SOLUTION}

Wo now consider the ganeral solution to the wquations (23) and (36) abeyed by the coefficients $E_{n}^{(i)}\left(x^{2}\right), F_{n}^{(i)}\left(x^{2}\right)$ oppeoring in the Wilson exponsion $(2)$. We First study the simplified equations which govern the Chang-fishbone colculation of Section III. A in which all self emergy correctlons, vertax corrections and ompittudes contoining a two peudo-scolar intermediate state hove been omitted. Naxt, those amplitudes contalning a two pseudo-scolor intermediate state are included and the resulting equations solved, In both ceses the functions $\tilde{E}_{n}^{(i)}\left(q^{2}\right)$ show a power depondence on $q^{2}$. where the exponent of $q^{2}$ depends explicitly on $n$. Thus for these examples the operators $O_{a_{1}}^{(t)} \ldots a_{n}$ possess en n-dependent enomalous dimension in the sense of Wilsen. Finally the general solution of eq. (40) for the vector theory is found, determining $\tilde{E}^{(0)}\left(q^{2}, 0\right)$ in terms of two unknown functions of a single vorioble and the quantitios $\beta(9), \gamma_{1}(0)$, $a_{n}^{i, j}(g)$. If wo astume that $B(g)$, hes a zero of $g=g_{\infty}$, ond that the quantitios $E_{n}^{(0)}\left(q^{2}, g\right)$ $\gamma_{1}(g)$ and $g_{n}^{t}(g)$ ore regulor of $g=g_{\infty}$, then this solution olso shows power dependence in $q^{2}$, with the power depending on $n$. Although in each of these three cases we find or hypothesize solutions which disploy a power behavior in $q^{2}$, we see no sughesrion that these powars should be identically zaro for all $n$ as is requirad if the strueture function $v W_{2}\left(q^{2}, u\right)$ is to be independent of $q^{2}$ for lorge $q^{2}$.

\section{A. CHANG FISHAANE AMPLITUDES}

Wo bogin by examining the of amplitudes first studied by Chang and Fithbone. These amplifudes contain no self energy corrections, no vertax corrections and no fntermediate stote composed of only two pesudo-scalor porticles. As was shown in Sect. IIJ. A. 
the resulting functions $\tilde{E}_{n}^{(1)}\left(q^{2}\right)$ have the form

$$
\tilde{E}_{n}^{(1)}\left(q^{2}\right)=r_{n} \times\left(q^{2}\right)^{-o_{n+2}^{1,1}}
$$

where the constants $v_{n}$ and $a_{n}^{1,1}+2$ can be computed in perturbotion theory:

$$
\begin{aligned}
v_{n} & =1+O\left(g^{2}\right) \\
a_{n+2}^{1,1}= & \frac{-\theta^{2}}{16 m^{2}(n+2)(n+3)}+O\left(g^{4}\right) .
\end{aligned}
$$

The position spoce function $E_{n}^{(0)}\left(x^{2}\right)$ follows from *\$. (80), (23) and (63) ${ }^{28}$;

$$
\begin{aligned}
E_{n}^{(1)}\left(x^{2}\right) & =-\frac{v_{n}+2}{2 n_{n+2}^{2} a_{n+2}} \frac{\Gamma\left(1-a_{n+2}^{1,1}\right)}{\Gamma\left(n+a_{n+2}^{1,1}+2\right)}\left(x^{2} / 4\right)^{a, 1} \\
& =\frac{v_{n+2}}{a_{n+2}^{1,1}}\left(x^{2}\right)^{a^{1,1}+2}
\end{aligned}
$$

where $\Gamma(z)$ is Euler's gommo function. A similar argument yields the longitudinal coefficients $F_{n}^{(1)}\left(x^{2}\right)$,

$$
F_{n}(1)\left(x^{2}\right)=\frac{v_{n}}{a_{n}^{1,1}-1}\left(x^{2}\right)^{a_{n}^{1,1}} .
$$

If these expressions are substituted into the Wilson exponsion (2), we find

$$
\begin{aligned}
J_{p}\left(\frac{x+y}{2}\right) J_{v}\left(\frac{-x+y}{2}\right)= & 4 \sum_{n=0}^{\infty}\left\{8^{\mu v} x_{a_{1}} \times a_{2}\left[\left(a_{n}^{1,1}-1\right) v_{n}-\left(a_{n}^{1} l_{1}+n-\frac{1}{2}\right) v_{n}^{\prime}\right]\right. \\
& \delta_{\mu a_{1} \delta_{v_{2}}} x^{2}\left[a_{n}^{1,1} v_{n}-\frac{1}{4} \frac{n(n-1)}{a_{n}^{1,1}-1} v_{n}^{m}\right]
\end{aligned}
$$


27

$$
\begin{aligned}
& -\left(x_{v} x_{a_{2}}{ }^{\delta} \mu a_{1}+x_{\mu} x_{a_{2}} \delta_{v a_{1}}\right)\left[\left(a_{n}^{1,1}-1\right) v_{n}+\frac{n}{2}, w_{n}\right] \\
& \left.-x_{\mu} x_{v} x_{a_{1}} x_{a_{2}}\left[\left(a_{n}^{1,1}-2\right) v_{n}^{\prime} \frac{1}{x_{2}}\right]\right\}\left(x^{2}\right)^{a_{n}^{1,1}} o_{a_{1}}^{(1)} \ldots a_{n} x_{a_{3}} \ldots x_{a_{n}}
\end{aligned}
$$

for $v_{0}=V_{1}=0$.

Thus if wo consider only amplitudes containing no self energy or vertex corrections and no two peavio-scalar intermediate states, the operators $O_{a_{1}}^{(1)} \ldots a_{n}$ possess on anomalous dimension $d_{n}$

$$
d_{n}=2+n+2 a_{n}^{1,1}
$$

in the sense of Wilson. Here $d_{n}$ is just the dimension(in units of mass) of the current $x$ current product on the left hand side of eq. (66), minus the dimension of the singular coefficient of the operator $\mathrm{O}_{a_{1}}^{(1)} \ldots a_{n}$ on the tight hand side of that equation. The dimension $d_{n}$ clearly depends on $n$ int o rather complicated way since to order $\theta^{2}$

$$
d_{n}=2+n-\frac{g^{2}}{8 n^{2} n(n+1)} \text {. }
$$

B. AMPLITUDES WITH SELF ENERGY AND VERTEX CORRECTIONS OMITTED

Next we study alt the amplitudes of the psoudo-scalar theory which do not contain self energy or vertex corrections. The resulting functions $\widetilde{E}_{n}^{(i)}(q)$ obey $\bullet q_{-}(27)$ with $\beta=\gamma_{1}=\gamma_{2}=0$. Thus

$$
-q^{2} \frac{\partial}{\partial q^{2}} \tilde{E}_{n}^{(1)}=a_{n+2}^{1,1} \tilde{E}_{n}^{(1)}+a_{n+2}^{1,2} \tilde{E}_{n}^{(2)}
$$




$$
-q^{2} \frac{\partial}{\partial q^{2}} \vec{E}_{n}^{(2)}=a_{n+2}^{2,1} \tilde{E}_{n}^{(1)}+a_{n+2}^{2,2} \vec{E}_{n}^{(2)}
$$

The general solutien to this set of coupled first order differential equations is

$$
\begin{aligned}
E_{n}^{(1)}\left(q^{2}\right)= & \psi_{n+2}^{(1)} \times\left(q^{2}\right)^{-v_{n}^{(1)}}+v_{n+2}^{(2)} \times\left(q^{2}\right)^{-v}(2) \\
E_{n}^{(2)}\left(q^{2}\right)= & -v_{n+2}^{(1)}\left(v_{n+2}^{(1)}+a_{n+2}^{1,1}\right) \frac{1}{a_{n+2}^{1,2}}\left(q^{2}\right)^{-v_{n+2}^{(1)}} \\
& -v_{n+2}^{(2)}\left(v_{n+2}^{(2)}+a_{n+2}^{1,1}\right) \frac{1}{a_{n+2}^{l, 2}}\left(q^{2}\right)^{-v_{n+2}^{(2)}}
\end{aligned}
$$

where the $v_{n}^{(j)}$ acte integralion constants and

$$
v_{n}^{(i)}=\frac{a_{n}^{1,1}+a_{n}^{2,2}}{2}+(21-3)\left[\frac{1}{4}\left(a_{n}^{1,1}-a_{n}^{2,2}\right)^{2}+a_{n}^{1,2} a_{n}^{2,1}\right]^{\frac{1}{2}}
$$

for $i=1,2$. As in the previous case, we can abtain the poition space functions $E_{n}^{(i)}\left(x^{2}\right)$ and $F_{n}^{(I)}\left(x^{2}\right)$ and substitute then into the Wilson exponsion (2), with the result

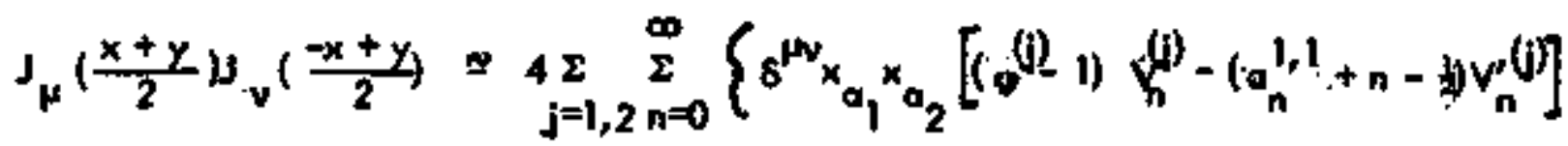

$$
\begin{aligned}
& +\delta_{\mu a_{1}}{ }^{v_{a_{2}}} x^{2}\left[v_{n}^{(j)} v_{n}^{(j)}-\frac{1}{4} \frac{n(n-1)}{\eta_{n}-1} v_{n}^{(j)}\right]^{\prime}
\end{aligned}
$$

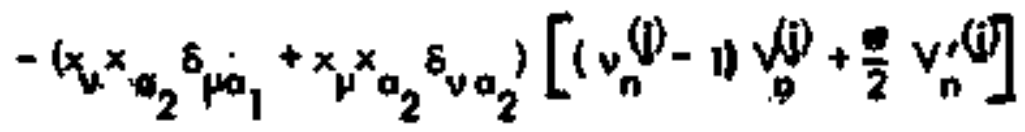

$$
\begin{aligned}
& \left.-x_{\mu} x_{v} x_{a_{1}} x_{a_{2}}(v)(j)-2 v_{n}^{\prime(j)} \frac{I}{x}_{x^{2}}\right\}\left(x^{2}\right)^{v(j)}
\end{aligned}
$$


29

$$
\left[o_{a_{1}}^{(1)} \ldots a_{n}^{(y)}-\frac{v_{n}^{(1)}+a_{n}^{l, 1}}{a_{n}^{1,2}} Q_{a_{1}}^{(2)} \ldots a_{n}(y)\right] \times a_{3} \ldots x_{a_{n}}
$$

Here the constants $v_{n}^{(i)}, v_{n}^{(i)}$ can be obtained from the $v_{n}^{(i)}, v_{n}^{(0)}$ by using eq. (8), where $v_{n}(j)$ is the integration constant multiplying $\left(q^{Z^{v}}{ }^{(j)}\right.$ in the expression for $F_{n}^{(i)}\left(q^{2}\right)$ analogous to 69. (70). Equation (72) implies that the operator

$$
\phi_{a_{1}}^{(1)} \ldots a_{n}-\frac{v_{n}^{(1)}+a_{n}^{1,1}}{a_{n}^{1,2}} o_{\alpha_{1}}^{(2)} \ldots a_{n}
$$

ho u anomalous dimension

$$
\underset{n}{(0)}=n+2+2 v(j)
$$

for $j=1,2$.

C. GENERAL SOLUTION

Finally we solve the exocet equation $(40)$ obeyed by the functions $E_{n}^{(0)}(q)$ in the vector theory. Eq. (40) can be rewritten as

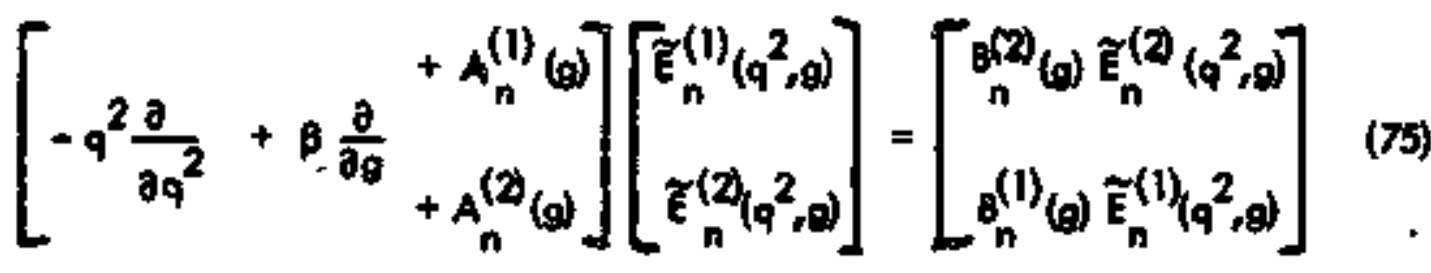

where $A_{n}^{(i)}$ and $B_{n}^{(i)}$ are liner combinations of $\gamma_{i}$ and $o_{n}^{i, k}$. Now define the new independent variables

$$
\begin{aligned}
& \rho(0)=\int_{\theta}^{\theta} \frac{d g^{\prime}}{\beta\left(g^{2}\right)} \\
& x\left(q^{2}, g\right)=\ln q^{2} / q_{0}^{2}+p(q)
\end{aligned}
$$


for some flxed values $g_{0}, q_{0}^{2}$. Let $G(\varphi)$ be the inverse of the function $p(9)$ deflned by eq. (760). In terms of these now voriables eq. (75) becomes

$$
\left[\begin{array}{ll}
\frac{\partial}{\partial p} & A_{n}^{(1)}(G(p)) \\
& +A_{n}^{(2)}(G(p))
\end{array}\right]\left[\begin{array}{c}
E_{n}^{(1)} \\
\widetilde{E}_{n}^{(2)}
\end{array}\right]=\left[\begin{array}{c}
\mathrm{B}_{n}^{(2)}(G(p)) \tilde{E}_{n}^{(2)} \\
B_{n}^{(1)}(G(p)) \tilde{E}_{n}^{(1)}
\end{array}\right]
$$

where the functions $\tilde{E}_{n}^{(i)}\left(q_{q}^{2} \exp (z-p), G(p)\right)$ are to be treated as function of $z$ and p. This wet of two first order couplad differential equations in the single variable $p$ has a general solution of the form

$$
\begin{aligned}
& \tilde{E}_{n}^{(1)}\left(q^{2}, g\right)=v_{n}^{(1)}\left(\ln q^{2} / q_{0}^{2}+\rho(g)\right) L_{n}^{(1)}(p(g))+v_{n}^{(2)}\left(\ln q^{2} / q^{2}+p(g)\right) \\
& L_{n}^{(2)}(p(\theta)) \\
& \widetilde{E}_{n}^{(2)}\left(q^{2}, \theta\right)=\frac{v_{n}^{(1)}\left(\ln q^{2} / q_{o}^{2}+\rho(\theta)\right)}{\theta_{n}^{(2)}(g)}\left[\frac{d}{d \rho} b_{n}^{(1)}(\rho(g))+A_{n}^{(1)}(g) L_{n}^{(1)}(\rho(\theta))\right] \\
& +\frac{v_{n}^{(2)}\left(\ln q^{2} / q_{0}^{2}+\rho(s)\right)}{B_{n}^{(2)}(g)}\left[\frac{d}{d p} L_{n}^{(2)}(p(g))+A_{n}^{(1)}(g) L_{n}^{(2)}(\rho(g))\right]
\end{aligned}
$$

where $v_{n}^{(1)}(z)$ and $v_{n}^{(2)}(z)$ are integration "constants" which con depend on $z=$ $\ln q^{2} / q_{0}^{2}+p(g)$ while $L_{n}^{(1)}(\rho)$ and $L_{n}^{(2)}(p)$ are the two independent solutions of the second order differential equation

$$
\begin{aligned}
& \left(\frac{d}{d p}+A_{n}^{(2)}(G(p))\right) \frac{1}{B_{n}^{(2)}(G(p))}\left(\frac{d}{d p}+A_{n}^{(1)}(G(p))\right) L_{n}^{(0)}(p) \\
& =B_{n}^{(1)}(G(p)) L_{n}^{(0)}(p)=0 .
\end{aligned}
$$


Thus the original equations (40) allow the two functions $\tilde{E}_{n}^{(i)}\left(q^{2}, g\right)$ which depend on two voriables to be datermined in terms of the two unknown functions $v_{n}^{(i)}(z)$ of a single veriable.

Now lot us speculate about a possible large $q^{2}$ behovior of the solutions

$\tilde{E}_{n}^{(i)}\left(q^{2}, a\right)$ given by $0 q .(78)$. Since the unknown functions $v_{n}^{(i)}(z)$ appoaring in $0 q .(78)$ depend only on the sum of $\ln q^{2} / q_{0}^{2}$ and $p(g)$, the large $q^{2}$ bahovior and the large $p$ behovior of the functions $\tilde{E}_{n}^{(1)}(q, G(p))$ are directly related once the large $p$ behavior of $A_{n}^{(i)}(G(p)), B_{n}^{(i)}(G(p))$ and $L_{n}^{(i)}(\rho)$ is known. In foct, carrying out the olgebroic steps outlined in Appendix D, we find:

$$
\tilde{E}_{n}^{(j)}\left(q^{2}, q\right)=\sum_{j=1,2} w_{n}^{j j}\left(\ln q^{2} / q_{o}^{2}+p(g), \theta\right) \tilde{E}_{n}^{(j)}\left(q_{q}^{2}, G\left(\ln q^{2} / q_{0}^{2}+p(\theta)\right)\right.
$$

where the quantitios $w_{n}^{i, j}(z, g)$, defined in Appondix $D$, are retional functions of $A_{n}^{(k)}$, $B_{n}^{(k)}$ and $L_{n}^{(k)}$. Thus the large $p$ bahavior of $\widetilde{E}_{n}^{(j)}\left(q_{o}^{2}, G(p)\right)$ determines, throwgh eq. (80), the large $q^{2}$ dependence of $\tilde{E}_{n}^{(i)}\left(q^{2}, g\right)$. Following $G$ ll-Mann and Low, we cansidior the possibility that $\beta(g)$ hes a rostc, $g_{\infty}$, so that

$$
\lim _{\boldsymbol{g} \rightarrow \mathbf{g}_{\infty}} \rho(g)=\infty
$$

If we ousume that the quentities $\tau_{n}^{(0)}\left(q_{0}^{2}, g\right)$ are woll defined and finite of the point $g=g_{\infty}$, then oq. (7g) dotermines the targe $q^{2}$ behovior of $\tilde{E}_{n}^{(1)}\left(q^{2}, g\right)$ in serms of the funetions $p(0), A_{n}^{(0)}(0)$ and $g_{n}^{(0)}(0)$ which oppear in our equation.

A particularly simplo asymptotic $q^{2}$ behavlor of $E_{n}^{(1)}\left(q^{2}\right)$ reaults if wo cosumbo that $g_{\infty}$ is a simple root of $\beta(g)$ and that $A_{n}^{(1)}(g)$ and $B_{n}^{(t)}(g)$ are repular at $g_{\infty}$. As is shown in Appendix D, these assumptions when combined with $\bullet 4$. (79) and (80) imply a simpla power behavior for $\tilde{E}_{n}^{(i)}\left(q^{2}\right)$. 


\section{CONCLUSIONS}

Uhing o technique of Symonzik and the Callon-Symanzlk equalion, we obtain o wrien of oquations obeyed to arbitrary onder in perturbotion theory by oll the c-number coefficients of the operetors apperring in the lifht cone exponsion of $J_{\mu}(x) J_{v}(0)$. These equations are used to dotermine the locodita logidsthmic behovior of $\checkmark W_{2}$ for two specific field theories, giving results in agreement with previous, more loborious calculotions. For simplified elosess of amplitudes in which no coupling constant renormolization is nequired, the equation predict a power low behovion of the confficionts $E_{n}^{(j)}\left(x^{2}\right)$ and a corrasponding anomalows dimansion $d_{n}=2+n+v_{n}$ for linear combinotions of the operoters $o_{a_{1}}^{(f)} \ldots a_{n}$ appeoring in the Wilson expansion. In genaral, the added quontity $v_{n}$ doponds in a non-trlitial fashion on $n$. Since the somo operators $o_{a_{1}}^{(i)} \ldots a_{n}$ enter both the transverse and longltudinal tesms In the Wilson expansion, the functions $E_{n}(i)\left(x^{2}\right)$ and $f_{n}(t)\left(x^{2}\right)$ both obey the same sel of equation. Thus, in this formalism only the prosence of difierent integrotion constants distingulates the small $x^{2}$ behovior of the traniverse and longltudinal components of the product $J_{j}(x) J_{v}(0)$. Finally, these equations allow us to speculate about the large $q^{2}$ and $v$ bohavior of $W_{1}$ and $W_{2}$, following the path proviously considered by Gall-Mom and Low, Wilson', and Symanzik". 
In this appendix we provide a derivation 12,29 of the Collen-Symanzik equations used in Sect. I. Let us begin by considering the pedo-sealer theory specifted by the Lograngion (13). The complete Logrongion, including counter terms is

$$
\begin{aligned}
& \mathcal{Y}=-\bar{\phi}\left(\gamma_{\mu} \partial_{\mu}+m\right) \phi-\frac{1}{2} a_{\mu} \phi a_{\mu} \phi-\frac{1}{2} \mu^{2} \phi^{2}+\frac{h}{4 !} \phi+i \sigma \phi \bar{\varphi} \gamma_{5} \phi \\
& -8 m Z_{2}-\frac{1}{2} \delta \mu^{2} Z_{3} \phi^{2}-\left(z_{2}-1\right) \bar{\phi}\left(\gamma_{\mu} \theta_{\mu}+m\right)_{\varphi}-\frac{1}{2}\left(Z_{3}-1\right) \times \\
& \left(a_{\mu} \phi a_{\mu} \phi+\mu^{2} \phi^{2}\right)+i\left(Z_{1}-1\right) \theta \phi \gamma_{\gamma_{5}}+\left(Z_{4}-1\right) \frac{h}{4 !} \phi^{4} .
\end{aligned}
$$

In order to spectfy the subtraction procedure represented by the above counter terms we consider the propogotors $S(\phi, m, \mu), \Delta\left(k^{2}, m, N\right)$ and the amputated vertex functions $\Gamma\left(p_{1}, P_{2}\right), \square\left(k_{1}, k_{2}, k_{3}\right)$ deffined by

$$
\begin{aligned}
& \left.S(\phi, m, \mu)=i \delta^{i p^{*} x} d^{4} x<0|\tau(\phi(0) \bar{\phi}(x))| 0\right\rangle \\
& \Delta\left(k^{2}, m, \mu\right)=i s^{i k \cdot x} d^{4} x<0|t(\phi(0) \mu(x))| 0> \\
& \left.\Gamma^{5}\left(p_{1}, p_{2}, m, \mu\right)=-i f \theta^{i\left(p_{1} x-p_{2} y\right)} d^{4} x d^{4} y<0|\tau(\omega(y) \phi(0) \bar{\sigma}(x))| i\right\rangle_{A} \\
& \square\left(k_{l}, k_{z}, k_{3^{\prime}} m, \mu\right)=-i f \theta^{i\left(k_{1}^{*} x+k_{2}^{*} y+k_{3}^{*} z\right)} d^{4} x d^{4} y d^{4} z \\
& <0|\mathrm{~T}(\phi(x) \phi(y) \phi(x))| 0\rangle_{A}
\end{aligned}
$$

The subtraction constents $Z_{1}, Z_{2}, Z_{3}, Z_{4}, 8 \mu^{2}$ and $6 m^{2}$ ore chosen so that the following conditions are satisfied: 


$$
\begin{aligned}
& S^{-1}(\phi, m, \mu)=0 ; \frac{\partial}{\partial \beta} s^{-1}(\beta)=-1 \text { ot } \beta=m \\
& \Delta^{-1}\left(k^{2}, m_{t} \mu\right)=0 ; \frac{\partial}{\partial k^{2}} \Delta^{-1}\left(k^{2}\right)=1 \text { of } k^{2}=-\mu^{2} \\
& \Gamma^{5}\left(p_{1}, p_{2}, m, \mu\right)=i \gamma_{5} g \text { at } \phi_{1}=p_{2}=m,\left(p_{1}-p_{2}\right)^{2}=-\mu^{2} \\
& \square\left(k_{1}, k_{2}, k_{3}, m_{,}\right)=h \text { of } k_{1}^{2}=k_{2}^{2}=k_{3}^{2}=-\mu^{2}, \\
& \left(k_{1}-k_{2}\right)^{2}=\left(k_{1}-k_{3}\right)^{2}=\left(k_{2}-k_{3}\right)^{2}=-\frac{4}{3} \mu^{2} .
\end{aligned}
$$

Having made this choice of subtraction constants we con now calculale order by arder in perturbation theory each Green's function $\Gamma\left(P_{1}, \ldots, P_{2 n}, k_{1}, \ldots k_{p}\right)$ for

$$
\begin{aligned}
& \Gamma\left(p_{1}, \ldots, p_{2 n}, k_{1}, \ldots, k_{p}\right)=\prod_{i=1}^{2 n} f d^{4} x_{i} \cdot i p_{i} \cdot x_{i} \underset{i=1}{p} \int d^{4} y_{i} e^{i} k_{i} \cdot y_{i} \\
& <0 \mid \tau\left(\psi\left(x_{1}\right) \ldots \phi\left(x_{n}\right) \bar{\psi}\left(x_{n+1}\right) \ldots \bar{\psi}\left(x_{2 n}\right)\right. \\
& \left.\phi\left(y_{1}\right) \ldots \phi\left(y_{p}\right)\right) \mid 0>
\end{aligned}
$$

as a function of $g, h, m$ and $\mu$.

In order to derive the Collan-Symanzik equations we consider a second proceduro for computing the Green's functions of this theory in which the subtractions are carried out at arbitrary points $\lambda_{1}$ and $\lambda_{2}$. Wo rewrite the Lograngion $\mathcal{Z}$ in torms of fields ${ }_{\lambda}, b_{\lambda}$ and coupling constants $\theta_{\lambda}, h_{\lambda}$ normalized ot these now points

$$
\psi=-\bar{\phi}_{\lambda}\left(\gamma_{\mu} \partial_{\mu}+m\right) \varphi_{\lambda}-\frac{1}{2} a_{\mu} \phi_{\lambda} a_{\mu} \phi_{\lambda}-\frac{1}{2} \mu^{2} \phi_{\lambda}^{2}+\frac{h_{\lambda}}{4 !} \phi_{\lambda}^{4}
$$


35

$$
\begin{aligned}
& +i g_{\lambda} \phi_{\lambda} \bar{\phi}_{\lambda} \gamma_{5} \phi_{\lambda}-\delta m z_{2, \lambda} \bar{\phi}_{\lambda} \phi_{\lambda}-\frac{1}{2} \delta \mu^{2} z_{3, \lambda} \phi^{2}-\left(z_{2, \lambda}-1\right) \phi_{\lambda} \times \\
& \left(\gamma_{\mu} \partial_{\mu}+m\right) \phi_{\lambda}-\frac{1}{2}\left(z_{3, \lambda}-1\right)\left(\phi_{\mu} \phi_{\lambda} a_{\mu} \phi_{\lambda}+\mu^{2} \phi_{\lambda}{ }^{2}\right)+i\left(z_{1, \lambda}-1\right) g_{\lambda} \phi_{\lambda} \phi_{\lambda} \gamma_{5} \phi_{\lambda} \\
& +\left(z_{4, \lambda}\right) \frac{h_{\lambda}}{4 !} \phi_{\lambda}^{4}
\end{aligned}
$$

The subtraction constants $z_{1, \lambda}, z_{2, \lambda}, z_{3, \lambda}, z_{4, \lambda}, 6 m, 8 \mu$ ore so chosen that the functions $s_{\lambda}, s_{\lambda}, r_{\lambda}^{5}$ and $\square_{\lambda}$ defined from eq. (A2) by replacing the fields + , 6 by $\phi_{\lambda}$, $\sigma_{\lambda}$ satisfy the following normalization conditions

$$
\begin{aligned}
& S_{\lambda}^{-1}(\phi, m, \mu)=0 \text { of } \not=m, \frac{\partial}{\partial \beta} s_{\lambda}^{-1}\left(\phi, \lambda_{1}, \lambda_{2}\right)=-1 \text { at } \phi=\lambda_{1} \\
& \text { (AGa) } \\
& \Delta_{\lambda}^{-1}\left(k^{2}, m, \mu\right)=0 \text { at } k^{2}=-\mu^{2}, \frac{\partial}{\partial k^{2}} \Delta_{\lambda}^{-1}\left(k^{2}, \lambda_{1}, \lambda_{2}\right)=1 \text { of } k^{2}=-\lambda_{2}^{2}
\end{aligned}
$$

(Abb)

$$
\begin{aligned}
& r_{\lambda}^{5}\left(p_{1}, p_{2}, \lambda_{1}, \lambda_{2}\right)=i \gamma_{5} g_{\lambda} \text { at } \phi_{1}=\phi_{2}=\lambda_{1},\left(p_{1}-p_{2}\right)^{2}=-\lambda_{2}^{2} \\
& \left.\square_{\lambda^{(} k_{1}, k_{2}}, k_{3}, \lambda_{1}, \lambda_{2}\right)=h_{\lambda} \text { of } k_{1}^{2}=k_{2}^{2}=k_{3}^{2}=-\lambda_{2}^{2} \text {, } \\
& \left(k_{1}-k_{2}\right)^{2}=\left(k_{1}-k_{3}\right)^{2}=\left(k_{2}-k_{3}\right)^{2}=-\frac{4}{3} \lambda_{2}^{2} \text {. }
\end{aligned}
$$

(Add)

The Lagraingians. . (A.) and (A5) are equal, the quantities $g_{\lambda}, h_{\lambda}$ being functions of $g, h, m, \mu, \lambda_{1}, \lambda_{2}$. The Greens functions $I_{\lambda}\left(p_{1}, \ldots, p_{2 n}, k_{1}, \ldots, k_{p}\right)$ computed by replacing the fields $\phi, 6$ by $\psi_{\lambda}, 6_{\lambda}$ in eq. (A4) are proportional 
to the original $r\left(p_{1}, \ldots, p_{2 n}, k_{1}, \ldots, k_{p}\right)$

$$
\left.\Gamma\left(p_{1}, \ldots, p_{2 n}, k_{1}, \ldots, k_{p}\right)=z_{2, \lambda}^{n} z_{3, \lambda}^{p / 2} r_{\lambda}^{\left(p_{1}\right.}, \ldots, p_{2 n}, k_{1}, \ldots, k_{p}\right)
$$

The Callon-Symanzik equations con be oblained by differentiating eq. (A7) with respect to $m$ and $\mu$ and then sotting $\lambda_{1}=m, \lambda_{2}=\mu$

$D_{\Gamma}=\left.\left\{n\left(D \dot{x}_{2, \lambda}\right)+\frac{P}{2}\left(D z_{3, \lambda}\right)+\left(D_{g_{\lambda}}\right) \frac{\partial}{\partial g_{\lambda}}+D h_{\lambda} \frac{\partial}{\partial h_{\lambda}}+m^{2} \frac{\partial}{\partial m^{2}}+\mu^{2} \frac{\partial}{\partial \mu^{2}}\right\} \Gamma_{\lambda}\right|_{\begin{array}{l}\lambda_{1}=m \\ \lambda_{2}=\mu\end{array}}$

$$
\text { for } D=m^{2} \frac{\partial}{\partial m^{2}}+\mu^{2} \frac{\partial}{\partial \mu^{2}} \text {. }
$$

When computed in perturbetion theory from the Lagrangion (A5), the Green's function $\Gamma_{\lambda}$ is determinad os a function of $g_{\lambda}, h_{\lambda}, m, \mu, \lambda_{1}, \lambda_{2}$; the lost two partial derivotives in $\theta q .(A \theta)$ of this function $I_{\lambda}, \partial / \partial m^{2}$ and $\partial / \partial \mu^{2}$, are to be performed with $g_{\lambda}$ and $h_{\lambda}$ hold fleed. This con be recognized as just the Callan-Symanzik Eg. (18), if we (o) identify

$$
\begin{aligned}
& \gamma_{1}=\frac{1}{2} D_{2, \lambda} \cdot Y_{2}=\frac{1}{2} D_{2, \lambda}, \beta *-D_{g_{\lambda}}, \beta^{\prime}=-D h_{\lambda} \\
& \Delta \Gamma=\left[m^{2} \frac{\partial}{\partial m^{2}}+\mu^{2} \frac{\partial}{\partial \mu^{2}}\right] \Gamma_{\lambda}
\end{aligned}
$$

all ovaluated at $\lambda_{1}=m, \lambda_{2}=\mu$; (b) sat $n=1, p=0$ or $n=0, p=2$; and (c) insert $J_{\mu}(x) J_{v}(0)$ into the time ordered product defining $\Gamma$ and $\Gamma_{\lambda}$. Wo need only show that the amplitude $\Delta \Gamma$ can be obtoined by inserting the mass operotor $U$ of Qq. (20) into the time ordered product (A4) defining $\Gamma$. Since only the mormalizotion 
condition for $s_{\lambda}$ and $\Delta_{\lambda}$ involve the mosses $m$ and $\mu$, the operotion $m^{2} \mathrm{a} / \mathrm{am}^{2}$ $+\mu^{2} \partial / \partial \mu^{2}$ when applied to the amplitude $I_{\lambda}$ yields a serios of toms, ooch obtained from $S_{\lambda}$ by (o) replocing a spinor propagator $S_{\lambda}(\phi)$ by

$$
-s_{\lambda}(\phi)\left\{\left[m^{2} \frac{\partial}{\partial m^{2}}+\mu^{2} \frac{\partial}{\partial \mu^{2}}\right] s_{\lambda}^{-1}(\phi)\right\} s_{\lambda}(\phi)
$$

or (b) replacing o pseudo-scalor propogotor $\Delta_{\lambda}^{\left(k^{2}\right)}$ by

$$
-\Delta_{\lambda}\left(k^{2}\right)\left\{\left[m^{2} \frac{\partial}{\partial m^{2}}+\mu^{2} \frac{\partial}{\partial \mu^{2}}\right] \Delta_{\lambda}^{-1}\left(k^{2}\right)\right\} \Delta_{\lambda}^{\left(k^{2}\right)} \text {. }
$$

On the other hond, the effect of inserting-iu into the time orderod product defining $\mathbf{r}$ is similor, yielding a sum of turms obtainad for $\Gamma$ by (o) roplacing a spinor propogator $S(p)$ by

$$
s_{0}^{1} p^{-} \times \delta^{4} x\left\langle 0|T[\phi(0) \cup \bar{\phi}(0)]|_{0} \equiv-S(\phi) U_{1}(\rho) S(\phi)\right.
$$

or (b) chonging a preudorscalor propogotor $\Delta\left(k^{2}\right)$ to

$$
\left.f \bullet^{i k \cdot x} d^{4} x<0|t[\phi(0) \cup \phi(x)]| 0\right\rangle \equiv-\Delta\left(k^{2}\right) u_{2}\left(k^{2}\right) \Delta\left(k^{2}\right)
$$

It is not difficult to tee that $U_{2}\left(k^{2}\right)$ and

$$
\left.\left(m^{2} \frac{\partial}{\partial m^{2}}+\mu^{2} \frac{\partial}{\partial \mu^{2}}\right) \Delta^{-1}\left(k^{2}\right)\right|_{\lambda_{1}=m, \lambda_{2}=\mu^{2}}
$$

obey Dyson integral equations with the same kernes. Since the normolization condition for $\partial / \partial k^{2} \Delta_{\lambda}^{-1}\left(k^{2}\right)$ in eq. (AGb) does not involve $m$ or $\mu, z_{3, \lambda}$ depends on $\theta_{\lambda}$ and $h_{\lambda}$ but not on $m$ or $\mu$. Thus the Dyson equations abeyed by both quantities contoin onty a constent inhomogenoous term. Therefore the two functions of $k^{2}$ must be 
30

proportional. If we let

$$
\delta_{2}=\left.\left[m^{2} \frac{\partial}{\partial m^{2}}+\mu^{2} \frac{\partial}{\partial \mu^{2}}\right] \Delta_{\lambda}^{-1}\left(k^{2}\right)\right|_{\lambda_{1}=m, \lambda_{2}=\mu}
$$

(A130)

and normalize the finite operators $N[\bar{\phi}(x) \psi(x)], N[f(x), f(x)]$ so that

$$
\begin{aligned}
& \left\langle p, s|N[\bar{\phi}(x) \phi(x)]|_{p, s}\right\rangle=\left\langle k|N[\phi(x) \phi(x)]|_{k}\right\rangle=1 \\
& \left\langle p, s|N[\phi(x) \phi(x)]|_{p, s}\right\rangle=\left\langle k|N[\bar{\psi}(x) \psi(x)]|_{k}\right\rangle=0 \quad \text { (A14) }
\end{aligned}
$$

where the state $\mid k>$ contains a single peudo-scolar particle of momentum $k$, then

$$
\left.\left[m^{2} \frac{\partial}{\partial m^{2}}+\mu^{2} \frac{\partial}{\partial \mu^{2}}\right] \Delta_{\lambda}^{-1}\left(k^{2}\right)\right|_{\substack{\lambda_{1} \\ \lambda_{2}=m}}=U_{2}\left(k^{2}\right) .
$$

(A15a)

Simitar arguments imply

$$
\left.\left[m^{2} \frac{\partial}{\partial m^{2}}+\mu^{2} \frac{\partial}{\partial \mu^{2}}\right] s_{\lambda}^{-1}(\beta)\right|_{\substack{\lambda_{1}=m \\ \lambda_{2}=\mu}}=u_{1}(\phi)
$$

if

$$
\delta_{1}=\left.2\left[m^{2} \frac{\partial}{\partial m^{2}}+\mu^{2} \frac{\partial}{\partial \mu^{2}}\right] s_{\lambda}^{-1}(\phi)\right|_{\substack{\lambda_{1}=m, \lambda_{2} \\ \phi=m}}
$$

(AlB)

Thus equation (APb) is justified and the Callon-5ymanzlk equations proved for the neutral peudo-scolar theory.

tet us now consider the vector theory. The complete Lagrangian, including 
39

counter terms, for this theory is

$$
\begin{aligned}
& \mathscr{P}=-\overline{4}\left(\gamma_{\mu} \partial_{\mu}+m\right) \phi-\frac{1}{4} G_{\mu \nu} G_{\mu \nu}-\frac{1}{2} \mu^{2} V_{\rho} V_{\rho}+I g V_{\mu} \bar{\phi} \gamma_{\mu} \\
& \left.-6 m z_{2} \bar{\psi} \psi-\frac{1}{2} 5 \mu^{2} z_{3} V_{\rho} V_{p}-\left(Z_{2}-1\right) \bar{\phi} \gamma_{\mu} \partial_{\mu}+m\right) \phi \\
& -\frac{1}{2}\left(Z_{3}-1\right)\left(\frac{1}{2} G_{\mu v} G_{\mu v}+\mu^{2} V_{p} V_{p}\right)+i\left(Z_{1}-1\right) \circ \bar{\phi} \gamma_{\mu} V_{\mu} . \quad \text { (All) }
\end{aligned}
$$

Introducing the propogoters ${ }^{30}$ and vertex functions

$$
\begin{aligned}
& \left.S(\phi, m, \mu)=t f^{i p \cdot x} d^{4} x<0|T(\mu(0) \bar{\psi}(x))| 0\right\rangle \\
& \Delta\left(k^{2}, m_{,}\right)\left(\sigma_{\mu v}-k_{\mu} k_{v} k^{2}\right)=i f *^{i k x} d^{4} x\left\langle 0\left|\tau\left(V_{\nu}(0) v_{v}(x)\right)\right| 0\right\rangle \\
& \Gamma_{\mu}\left(p_{1}, p_{2}, m, \mu\right)=-i f e^{i p_{1} \cdot x-i p_{2} \cdot y} d^{4} x d^{4} y \\
& \left\langle 0\left|T\left(\psi(y) \vee_{\mu}(0) \bar{\psi}(x)\right)\right| 0\right\rangle
\end{aligned}
$$

we choose the subtraction constants $Z_{1}, Z_{2}, Z_{3}, 6 m, 8 \mu^{2}$ so that

$$
\begin{aligned}
& \left.s^{-1}(\phi, m, \mu)\right|_{\beta=m}=0 ;\left.\frac{\partial}{\partial \beta} s^{-1}(\phi, m, \mu)\right|_{\alpha=m}=-j \\
& \left.\Delta^{-1}\left(k^{2}, m, \mu\right)\right|_{k^{2}=-\mu^{2}}=0 ;\left.\left.\frac{\partial}{\partial k^{2}} \Delta^{-1}\left(k^{2}, m, \mu\right)\right|_{k}\right|_{-\mu^{2}}=1 \\
& \left.\Gamma\left(p_{1}, p_{2}, m, \mu\right)\right|_{\phi_{1}=\beta_{2}=m}=i g \gamma_{\mu} . \\
& \left(p_{1}-p_{2}\right)^{2}=-\mu^{2}
\end{aligned}
$$

(AlB)

The electromagnetic interaction of the charged spine field is now included by adding the interaction term 
40

$$
\begin{aligned}
\partial \gamma= & +i \odot A_{\mu}-\gamma_{\mu} \phi+\frac{1}{2} f F_{\mu \nu} G_{\mu \nu}+\frac{1}{2} \delta f Z_{3}^{\frac{1}{2}} F_{\mu \nu} G_{\mu \nu}+\frac{1}{2} f\left(Z_{3}^{\frac{1}{2}}-1\right) F_{\mu \nu} G_{\mu \nu} \\
& +i \oplus\left(Z_{1}-1\right) A_{\mu} \phi \gamma_{\mu} \phi
\end{aligned}
$$

where the subtraction constant $5 f$ is 30 defined that

$$
\left.\Delta_{1}\left(k^{2}, m, \mu\right)\right|_{k^{2}=-k^{2}}=f \mu^{2}
$$

for

$$
\begin{aligned}
\Delta_{1}\left(k^{2}, m, \mu\right)\left[\delta_{\mu v}-k_{\mu v} k_{v} / k^{2}\right]= & i f e^{i k \cdot x} d^{4} x \\
& \left\langle 0\left|T\left(v_{\mu}(0) A_{v}(x)\right)\right| 0\right\rangle_{A} .
\end{aligned}
$$

(ADO)

We hove not included counter terms of order $e^{2}$ or higher in eq. (A19). Just as in the preudo-scolor cost we can consider fields $Y_{\lambda}, V_{\lambda}$ and coupling constants $9_{\lambda}$, $f_{\lambda}$ defined according to a second normalization scheme:

$$
\begin{aligned}
& \left.s_{\lambda}^{-1}(\phi, m, \mu)\right|_{\beta=m}=0 ;\left.\quad \frac{\partial S^{-1}}{\partial \phi}\left(\phi, \lambda_{1}, \lambda_{2}\right)\right|_{\beta=\lambda_{1}}=-1 \\
& \left.\Delta_{\lambda}^{-1}\left(k^{2}, m, \mu\right)\right|_{k=-\mu^{2}}=0 ;\left.\frac{\partial \Delta_{\lambda}^{-1}}{\partial k^{2}}\left(k^{2}, \lambda_{1}, \lambda_{2}\right)\right|_{k}{ }^{2}=-\lambda_{2}^{2}=1 \\
& \left.\Gamma_{\lambda_{\nu} \mu}\left(p_{1}, p_{2}, \lambda_{1}, \lambda_{2}\right)\right|_{\phi_{1}=\phi_{2}=\lambda_{1}}=i g_{\lambda} \gamma_{\mu} \\
& \left(p_{1}-p_{2}\right)^{2}=-\lambda_{2}^{2} \\
& A_{1},\left.\lambda^{\left(k^{2}, \lambda_{1}, \lambda_{2}\right)}\right|_{k}{ }_{k=-\lambda_{2}^{2}}=f_{\lambda} \lambda_{2}^{2} \text {. }
\end{aligned}
$$

(A21o)

(A lc)

(A21d) 
41

Differentiating eq. (A7), rewritten for the vector case, we find

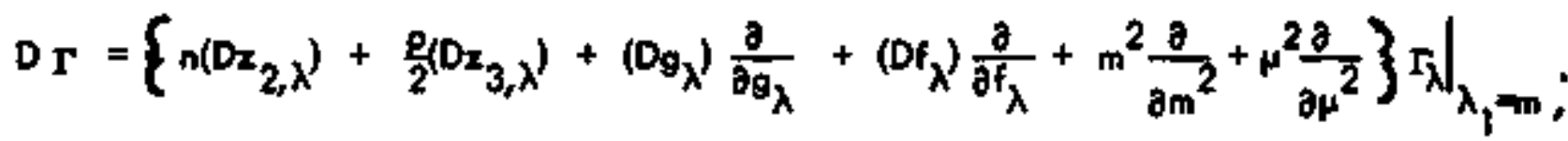

$$
\begin{aligned}
& \lambda_{2}=
\end{aligned}
$$

(A22)

This is the complete Collon-Symanzik equation obeyed by the amputated time ordered product of in spinor fields, $p$ vector fields and $r$ electromognelle currents,

$$
U_{\mu}=\partial_{v} F_{v \mu}
$$

The quantities $\mathrm{Dz}_{2, \lambda}, \mathrm{Dz}_{3, \lambda}$ and $\mathrm{Dg}_{\lambda}$ can be identified with $\gamma_{1}, \gamma_{2}$ and $\rightarrow$ of eq. (33) respectively while an argument similar to that given in the proceeding pseudoscalar case shows that

$$
\vec{\gamma}_{3} \Delta \Gamma=\left(m^{2} \frac{\partial}{\partial m^{2}}+\mu^{2} \frac{\partial}{\partial \mu^{2}}\right) \Gamma_{\lambda} l_{\lambda_{1}=m, \lambda_{2}=\mu} \cdot
$$

Finally we can directly compute Of $_{\lambda}$ by noting that $\Lambda_{(}\left(k^{2}, m, \mu\right)$ and $1 / 9 \Delta^{-1}$ $\left(k^{2}, m, \mu\right)$ can, by definition, differ only by a first degree polynomial in $k^{2}$ so that

Qq. (A20) and current conservation $\left(\Delta_{1}(0, m, \mu)=0\right)$ implies

$$
\Delta_{1}\left(k^{2}, m, \mu\right)=-f k^{2}+\frac{k^{2}+\mu^{2}}{\mu^{2}} \frac{1}{g} \Delta^{-1}(0, m, \mu)+\frac{1}{g} \Delta^{-1}\left(k^{2}, m, \mu\right) .
$$

Likewise

$$
\begin{aligned}
\left.\Delta_{1, \lambda}{ }^{2}, m, \mu\right)= & -f_{\lambda} k^{2}-\frac{k^{2}}{\lambda_{2}} \frac{1}{\theta_{\lambda}} \Delta_{\lambda}^{-1}\left(0, \lambda_{1}, \lambda_{2}\right)-\frac{1}{g_{\lambda}} \Delta_{\lambda}^{-1}(0, m, \mu) \\
& +\frac{1}{g_{\lambda}} \Delta_{\lambda}^{-1}\left(k^{2}, m, \mu\right)
\end{aligned}
$$


42

where the coefficient of $k^{2}$ is guaranteed by ow subtraction procedure to be independent of $m$ and $\mu$ for fixed $f_{\lambda}$ and is therefore determined by the condition (A21d). In analogy with eq. (A7) of the pseudo-scalcr case we hove

$$
\begin{aligned}
& \Delta_{1, \lambda}\left(k^{2}, m, \mu\right)=z_{3, \lambda}^{-\frac{1}{2}} \Delta_{1}\left(k^{2}, m, \mu\right) \\
& \left.\Delta_{\lambda}^{-1}\left(k^{2}, m, \mu\right)=z_{3, \lambda^{-1}}^{-1} k^{2}, m, \mu\right) \\
& s_{\lambda}^{-1}(\phi, m, \mu)=z_{2, \lambda}^{-1} s^{-1}(\phi, m, \mu) \\
& T_{\lambda, \mu}\left(p_{1}, p_{2}, m, \mu\right)=z_{3, \lambda}^{-\frac{1}{2}} z_{2, \lambda}^{-1} \Gamma_{j}\left(p_{1}, p_{2}, m, \mu\right) .
\end{aligned}
$$

(A260)

(A266)

(A26e)

(ARd)

Es. (A26C) and (A26d) together with the Ward identities

$$
\begin{aligned}
& \frac{1}{\theta}\left(p_{1}-p_{2}\right)_{\mu} F_{\mu}\left(p_{1}, p_{2}\right)=s^{-1}\left(p_{1}\right)-s^{-1}\left(p_{2}\right) \\
& \frac{1}{g_{\lambda}}\left(p_{1}-p_{2}\right)_{\mu} \Gamma_{\lambda, \mu}\left(p_{1}, p_{2}\right)=s_{\lambda}^{-1}\left(p_{L}\right)-s_{\lambda}^{-1}\left(p_{2}\right),
\end{aligned}
$$

implied by current conservation and our normalization procedures (A18) and (A21), yield

$$
\mathbf{g}_{\lambda}=z_{3, \lambda}^{-\frac{1}{2}} \mathbf{g}
$$

Combining eq. (A25), (A26a), (A266) and (A28) we obtain

$$
f_{\lambda}=z_{3, \lambda}^{-\frac{1}{2}} f-\frac{1}{9} \frac{\Delta_{\lambda}^{-1}\left(0, \lambda_{1}, \lambda_{2}\right)}{\lambda_{2}^{2}}+\frac{1}{9} z_{3, \lambda}^{-\frac{1}{2}} \frac{\Delta^{-1}(0, m, \mu)}{\mu^{2}},
$$

or

$$
D f_{\lambda_{1}=m, \lambda_{2}=\mu}=-\gamma_{2}^{f}+\left[\beta \frac{\partial}{\partial g}-\gamma_{2}\right] \frac{1}{g} \frac{\Delta^{-1}(0, m, \mu)}{\mu^{2}} ;
$$


43

so that our complete Callan-Symonzik equation rads

$$
\begin{aligned}
& \left\{m^{2} \frac{\partial}{\partial m^{2}}+\mu^{2} \frac{\partial}{\partial \mu^{2}}+\beta \frac{\partial}{\partial g}-2 m \gamma_{1}-p \gamma_{2}\right. \\
& \left.+\left[\gamma_{2} f-\left(\beta \frac{\partial}{\partial g}-\gamma_{2}\right) \frac{1}{g} \frac{\Delta^{-1}(0, m, \mu)}{\mu^{2}}\right] \frac{\partial}{\partial f}\right\} r=\gamma_{3}^{\top} \Delta \Gamma .
\end{aligned}
$$

If $\Gamma$ is computed to lowest order in $\theta$, the dependence on $f$ is known, allowing the partial derivative with respect to $f$ in eq. (A31) to be explicitly carried out. If wo assume that each electromagnetic current carries a momentum transfer squared much greater than $\mu^{2}$, then eq. (A250) implies that if each current $J_{\mu}(x)$ is replaced by

$$
\left[f+\frac{1}{g \mu^{2}} \Delta^{-1}(0, m, \mu)\right] V_{\mu}(x) \text {, }
$$

- the Green's function $\Gamma$ is not changed. Thus

$$
\left[\theta^{f}+\frac{1}{\mu^{2}} \Delta^{-1}\left(0, m, \mu^{2}\right)\right]^{-r} r
$$

is Independent of $f$ and eq. (A3)) can be rewritten

$$
\begin{aligned}
& \left\{m^{2} \frac{\partial}{\partial m^{2}}+\mu^{2} \frac{\partial}{\partial \mu^{2}}+\rho \frac{\partial}{\partial \theta}-2 n \gamma_{1}-p \gamma_{2}+2 \gamma_{2}\right)\left(g f+\frac{1}{\mu^{2}} \Delta^{-1}(0, m, \mu)\right)+\Gamma \\
& \Rightarrow \Delta r
\end{aligned}
$$

for

$$
\gamma_{3}=\left[g f+\frac{1}{\mu^{2}} \Delta^{-1}(0, m, \mu)\right]^{-1}
$$

which is just eq. (33). In obtaining the form (A32) we have used the relationship 
$\beta=8 \gamma_{2}$

(A34)

implied by *q. (A2B). 


\section{APPENDIX B}

In this appendix a detailad calculation of $a_{n}^{1,1}$ to order $g^{2}$ is presented for the pseudo-scalor theory. Recall that

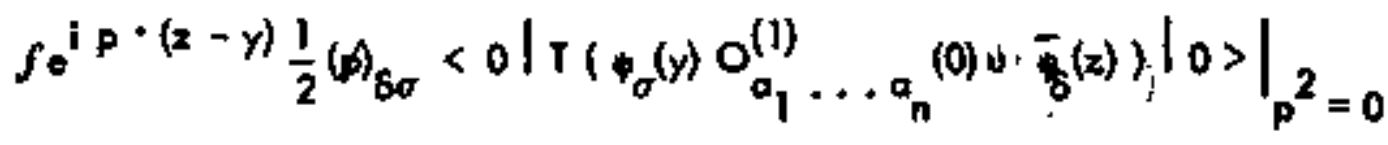

$$
\begin{aligned}
& =(i)^{n}-a_{n}^{1,1} P_{o_{1}} \cdots: P_{a_{n}}+\left(\text { terms coritaining } \delta_{a_{j}} a_{j}\right)
\end{aligned}
$$

for $1 \leq \alpha_{1} \leq 3$. Since to lowest order in $g^{2}$ no counter terms must be added to moke the operator $o_{a_{1}}^{(1)} \ldots a_{n}$ finite,

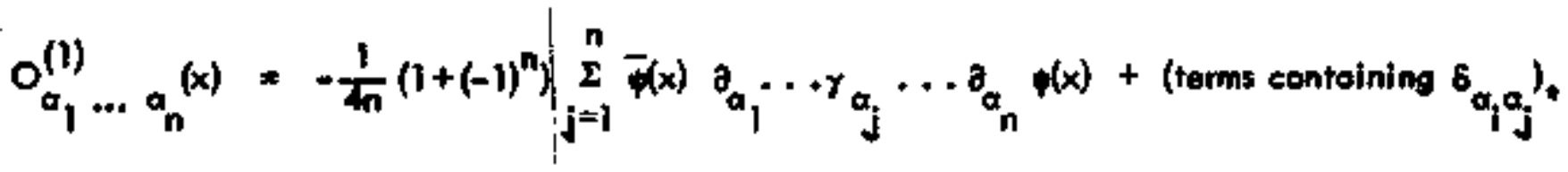

Figure (2a) illustrates the three graphs contributing to $a_{n}^{1, t}$ to order $g^{2}$. In foct, to order $g^{2}, a_{n}^{1,1}$ requires no renormolizotion of any sort, aither within the operator $O_{\alpha_{1}}^{(1)} \ldots a_{n}$ or of propagators or other vertices. Consequently, the effect of the operotor $\checkmark$ is simply to differentiate the order $g^{2}$ matrix element of $O_{a_{1}}^{(1)} \ldots a_{n}$ represented by figure $2 \mathrm{~b}$ with respect to the internal mosses:

$$
\begin{aligned}
& a_{n}^{t, 1} p_{a_{1}} \ldots p_{a_{n}} \\
& =\frac{1}{4 n} \sum_{j=1}^{n} \frac{g^{2}}{(2+)^{4}} \int d^{4} k\left[m^{2} \frac{\partial}{\partial m^{2}}+\mu^{2} \frac{\partial}{\partial \mu^{2}}\right]^{n} \frac{\left(f(k+m)\left(k_{a_{1}} \cdots \gamma_{a_{j}} \cdots k_{a_{n}}\right)(K+m)\right.}{\left.\left[k^{2}+m^{2}-i k\right]^{2}(k-p)^{2}+\mu^{2}-i a\right]^{2}} \\
& + \text { (tarms contoining } b_{a_{i} \alpha_{j}} \text { ) }
\end{aligned}
$$


46

for $p^{2}=0$. (The quantities $\delta_{1}$ and $\delta_{2}$ appearing in the definition (20) of $u$ are both unity to lowest order in g.) It is useful to observe that the mass terms in the numerator do not contribute to the $\mathrm{P}_{\alpha_{1}} \ldots P_{a_{n}}$ termstince. if the differentiation $m^{2} \partial / \partial m^{2}+\mu^{2} a / \partial \mu^{2}$ were performed after the integration over $k$, then upon integration such terms would yield finite functions of $\mu^{2} / \mathrm{m}^{2}$ which would bo annihilated by the derivatives.

The $P_{a_{1}}, \ldots p_{a_{n}}$ term in the above integral con be easily evaluated if the integration variables are changed to those of Sudakov. Let.

$$
P=(0,0, P, I P, \bar{P}=(0,0,+P,-i P)
$$

and

$$
k=a \dot{p}+9 \dot{p}+k
$$

for:

$$
k_{1}=\left(k_{1}, k_{2}, 0,0\right)
$$

In terms of the variables $a, \beta, k_{1}, k_{2}, e q$. (BS) becomes.

$$
\begin{aligned}
a_{n}^{1,1}= & \frac{2 p^{2} q^{2}}{(2 n)^{4}} \int_{-\infty}^{\infty} d a \int_{-\infty}^{\infty} f \beta f d^{2} k\left[m^{2} \frac{a}{\partial m^{2}}+\mu^{2} \frac{\partial}{\partial \mu^{2}}\right] \\
& {\left[4 p^{2} \alpha \beta+k_{1}^{2}+m^{2}-i \epsilon\right]^{2}\left[4 p^{2}(\beta-1)+k_{1}^{2}+p^{2}-i \epsilon\right] }
\end{aligned}
$$

where we have equated the coefficients of ${p_{a_{1}}}_{1} \ldots p_{a_{n}}$ and evaluated the trace in $4 q$. (B3) according to

$$
n\left(\alpha k \gamma_{o_{1}} k\right)=-4 i k_{1}^{2} p_{a_{i}}+\left(\text { terms with } \vec{p}_{a_{j}} \text { or }\left(k_{1}\right)_{\alpha_{i}}\right. \text {. }
$$


The integral over a ean be performed using contour integration so that

$$
\begin{aligned}
a_{n}^{1,1}= & \frac{g^{2}}{2(2 \pi)^{3}} \int_{0}^{1} d \beta \int \partial^{2} k\left[m^{2} \frac{\partial}{\partial m^{2}}+\mu^{2} \frac{\partial}{\partial \mu^{2}}\right] \\
& \frac{(1-\beta) \beta^{n-1} k_{1}^{2}}{\left[\beta \mu^{2}+(1-\beta) m^{2}+k_{\perp}^{2}\right]^{2}} \\
= & -\frac{1}{16 \pi^{2}} \int_{0}^{1} d \beta \beta^{n-1}(1-\beta)=-\frac{1}{16 \pi^{2}} \frac{1}{n(n+1)} .
\end{aligned}
$$




\section{APPENDIX C}

We now give the results of a colculation of all the constenis $a_{n}^{i j j}$ to order $\theta^{2}$ in both the pacudo-scolor and vector theories. The values of $a_{n}^{i, j}$ found for the paeudocealar theory are shown in figure 3. Also shown are feyaman dlograms representing those amplifudes which when differentiated with respoct to the internol messes give the odjocent values of $a_{n}^{t / j}$. The vertex joining two photon lines and we formion lines represents the foctor

$$
-\frac{1}{2} \frac{1}{n} \sum_{j=1}^{n} P_{a_{1}} \cdots r_{a_{j}} \cdots P_{a_{n}}
$$

in the correuponding Feymon omplitudes, where is is the four-momentum corried by the incoming formion line. Likewise, the vertax connecting two photon and two psoudoscolor lines represents the faetor

$$
k_{a_{1}} \cdots k_{a_{n}}
$$

whore $k$ ts the momentum carried by one of the proudo-scalor porticles.

The resuls in the vector theory, shown with their corresponding grophs in fig. 4, ore somewhot more complicated. The pressonet of the vector flelds $V_{a_{1}}$ in the operator $0_{a_{1}}^{(1)} \ldots a_{n}$ defined in eq. (37a) implies that this operotor not only cantributes the two photon - two fermion vertox found in fig. 4, representing the foctor (C1), but olis giveso two photon - no formion - vector partielo vartox contrlbuting to $a_{n}^{1,1}$ and $a_{n}^{2,1}$. This vertex mepresents the foctor

$$
\frac{i g}{2 \pi(n-1)} \sum_{\substack { l=1 \\
\begin{subarray}{c}{j=1 \\
j \neq l{ l = 1 \\
\begin{subarray} { c } { j = 1 \\
j \neq l } }\end{subarray}}^{n}(p+k) a_{1} \cdots(p+k) a_{l-1} \delta \sigma_{l} p_{a_{l+1}} \cdots \gamma_{a_{j}} \cdots p_{a_{n}}
$$


49

where $p$ and $k$ are the momenta carried in by the spinet and vector particles raspertimely while . $p$ is the vector particle's polarization index. Finally the two photon - two vector particle vertex in fig. 4 represents the factor

$$
\begin{aligned}
& \frac{3}{2 \pi(n-1)} \sum_{\substack{l=1 \\
j=1 \\
j \neq l}}^{n}\left(k_{a_{1}} \cdots \delta_{a_{l}} \cdots \delta_{a_{j}} \cdots k_{a_{n}}\right) \\
& \left(k_{\mu v} k_{v} \delta_{\sigma \rho}+k_{\rho \psi}^{2} \delta_{\sigma \nu}^{\delta}-k_{p} k_{v} \delta_{\sigma \mu}-k_{\sigma} k_{v} \delta_{\rho \mu}\right)
\end{aligned}
$$

where $k$ is the momentum carried by the vector line and $\rho, \sigma$ the vector particles' polarization indican. 


\section{APPENDIX D}

Finolly, we investigate the large $q^{2}$ behovior of the solutions (78) to eq. (75). First the lorge $q^{2}$ limit of $Z_{n}^{(i)}(q, g)$ is related to the large $p$ limit of $A_{n}^{(j)}(G(p))$, $\mathrm{g}_{n}^{(i)}(G(p))$ and $\widetilde{E}_{n}^{(i)}\left(q_{0}^{2}, G(p)\right)$. Then we consider the possibllity, first Identified by Gell-Mann and Low, that the function $\beta(g)$ has a zero at $g=g_{\infty}$. In that case, if $\widetilde{E}_{n}\left(q^{2}, g\right)$ is weil defined and nan-zero of $g=g_{\infty}$, then the asymptosic behavior of $\widetilde{E}_{n}^{(i)}\left(q{ }^{2}, g\right)$ for lorge $q^{2}$ is determined by the functions $A_{n}^{(i)}(g), B_{n}^{(i)}(g)$ and $p(g)$. If in addition $g_{\infty}$ is a simple zero of $\beta$ and $A_{n}^{(i)}(g), B_{n}^{(i)}(g)$ are regular at $g_{\infty}$ then a power behovior in $q^{2}$ for $\widetilde{E}_{n}^{(i)}\left(q^{2}, g\right)$ is deduced for lorge $q^{2}$.

First, $q^{2}$ is reploced by $q_{0}^{2}$ in $0 q .(78)$ so that $z=p(g)$, or $g=G(z)$, and the resuliting equation solved for the functions $v_{n}^{(i)}(z)$ :

$$
\begin{aligned}
v_{n}^{(1)}(z)= & \frac{1}{w(z)}\left\{\left[\frac{d}{d z} L_{n}^{(2)}(z)+A_{n}^{(1)}(G(z)) L_{n}^{(2)}(z)\right] E_{n}^{(1)}\left(q_{0}^{2}, G(z)\right)\right. \\
& \left.-B_{n}^{(2)}(G(z)) L_{n}^{(2)}(z) E_{n}^{(2)}\left(q_{0}^{2}, G(z)\right)\right\} \\
v_{n}^{(2)}(z)= & -\frac{1}{w(z)}\left\{\left[\frac{d}{d z} L_{n}^{(1)}(z)+A_{n}^{(1)}(G(z)) L_{n}^{(1)}(z)\right] E_{n}^{(1)}\left(q_{0}^{2}, G(z)\right)\right. \\
& \left.-B_{n}^{(2)}(G(z)) L_{n}^{(1)}(z) E_{n}^{(2)}\left(q_{0}^{2}, G(z)\right)\right\}
\end{aligned}
$$

where

$$
w(z)=\left[\frac{d}{d z} L_{n}^{(2)}(z)\right] L_{n}^{(1)}(z)-\left[\frac{d}{d z} L_{n}^{(I)}(z)\right] L_{n}^{(2)}(z) .
$$

The equations can now be used to eliminate the functions. $v_{n}^{(i)}(z)$ from 0 ..$(78)$, yielding 
5)

an expression for $\tilde{E}_{n}^{(i)}\left(q^{2}, g\right)$ in terms of $E_{n}^{(i)}\left(q_{o}^{2}, G\left(\ln \left(q^{2} / q_{0}^{2}\right)+\rho(g)\right)\right)$ so that the large $q^{2}$ and the large $p$ behavior of $\tilde{E}_{n}^{(i)}\left(q^{2}, G(p)\right)$ are related:

$$
\tilde{E}_{n}^{(i)}\left(q^{2}, \theta\right)=\sum_{i=1,2} w_{n}^{i, i}(z, \theta) \widetilde{E}_{n}^{(j)}\left(q_{0}^{2}, G(z)\right)
$$

for $z=\ln q^{2} / q_{0}^{2}+\rho(\rho)$ and

$$
\begin{aligned}
& w_{n}^{1,1}(z, g)=\frac{1}{w(z)}\left\{\left[\frac{d}{d z} L_{n}^{(2)}(z)+A_{n}^{(1)}(G(z)) L_{n}^{(2)}(z)\right] L_{n}^{(1)}(p(g))\right. \\
& \left.-\left[\frac{d}{d z} L_{n}^{(1)}(z)+A_{n}^{(1)}(G(z)) L_{n}^{(1)}(z)\right] L_{n}^{(2)}(\rho(g))\right\} \\
& w_{n}^{l, 2}(z, g)=\frac{1}{w(z)}\left\{L_{n}^{(1)}(z) L_{n}^{(2)}(\rho(0))-L_{n}^{(2)}(z) L_{n}^{(1)}(\rho(\theta))\right\} B_{n}^{(2)}(G(z)) \\
& w_{n}^{2,1}(z, g)=\frac{1}{w(z)}\left([ \frac { d } { d z } L _ { n } ^ { ( 2 ) } ( z ) + A _ { n } ^ { ( 1 ) } ( G ( z ) ) L _ { n } ^ { ( 2 ) } ( z ) ] \left[\frac{d}{d \rho} L_{n}^{(1)}(\rho(\rho))\right.\right. \\
& \left.+A_{n}^{(1)}(g) L_{n}^{(1)}(\rho(g))\right]=\left[\frac{d}{d z} L_{n}^{(1)}(z)+A_{n}^{(1)}(G(z)) L_{n}^{(1)}(z)\right] \\
& \left.\left[\frac{d}{d_{\rho}} L_{n}^{(2)}(\rho(g))+A_{n}^{(1)}(g) L_{n}^{(2)}(p(o))\right]\right\}\left[B_{n}^{(2)}(g)\right]-1 \\
& w_{n}^{2,2}(z, 0)=\frac{a_{n}^{(2)}(G(z))}{B_{n}^{(2)}(g)} \frac{w(g(g))}{w(z)} w_{n}^{1,1}(p(g), G(z)) .
\end{aligned}
$$

If wo assume that $\beta(g)$ has o root $g_{\phi}$ and that $\tilde{E}_{n}^{(i)}\left(q_{0}^{2}, g\right)$ is regular and nonzero of $g=\theta_{\infty}$, then eq. (D3) determines the asymptotic form of $F_{n}^{(i)}\left(q^{2}, g\right)$, for $g<q_{\infty}$, in terms of the functions $A_{n}^{(i)}(g), g_{n}^{(j)}(g)$ and $p(g)$ appearing in our eq. (75). In particular, if we assume that $A_{n}^{(j)}(g), B_{n}^{(i)}(g)$ are regular at $g_{\infty}$ and that $g_{\infty}$ is a 
simple zero of $\beta(a)$, then o power behovior for $\tilde{E}_{n}^{(i)}(q)$ is implied by eq. (D3) ${ }^{31}$. In order to show this, we must determine the large $z$ behavior of $L_{n}^{(f)}(z)$ and hance of $w_{n}^{j}, i_{(}(z, g)$. It is not difficult to see from $\bullet q .(79)$ that, under these conditions on $A_{n}^{\prime}(g)$, $a_{n}^{i}(g)$ and $p(g)$, the functions $L_{n}^{(i)}(z)$ can be so ehosen that

$$
L_{n}^{(i)}(\ln y) \sim y^{v_{1}}\left[1+O\left(\frac{1}{y}\right)\right]
$$

for $y$ lorge and

$$
\begin{aligned}
v_{n}^{(i)}= & -\frac{1}{2}\left(A_{n}^{(1)}\left(g_{\infty}\right)+A_{n}^{(2)}\left(g_{\infty}\right)\right)+(2 i-3)\left[\frac{1}{4}\left(A_{n}^{(1)}\left(g_{\infty}\right)-A_{n}^{(2)}\left(o_{\infty}\right)\right)^{2}\right. \\
& \left.+g_{n}^{(1)}\left(g_{\infty}\right) B_{n}^{(2)}\left(g_{\infty}\right)\right]^{\frac{1}{2}} .
\end{aligned}
$$

This asymptotic form for $L_{n}^{(i)}(z)$ con then be substitutiod into eq. (D3) yielding

$$
\begin{aligned}
& \widetilde{E}_{n}^{(1)}\left(q^{2}, g\right)=\sum_{i=1,2} \sigma_{n}^{(i)} B_{n}^{(2)}(g) L_{n}^{(i)}(p(\theta))(q)^{-v} \cdot n \\
& \widetilde{E}_{n}^{(2)}\left(q^{2}, g\right)=\sum_{j=1,2} \sigma_{n}^{(i)}\left[\frac{d}{d p} L_{n}^{(0)}(p(\theta))+A_{n}^{(0)}(g) L_{n}^{(i)}(p(g))\right]\left(q^{2}\right)^{-v_{n}^{(i)}}(
\end{aligned}
$$

for

$$
q_{n}^{(1)}=\frac{\left(q_{o}^{2}\right)_{n}^{(1)}}{e^{p(o) v_{n}^{(1)}} \frac{B_{n}^{(2)}\left(g_{\infty}\right) E_{n}^{(2)}\left(q_{o}^{2}, g_{\infty}\right)-\left[v_{n}^{(2)}+A_{n}^{(1)}\left(g_{\infty}\right)\right] E_{n}^{(1)}\left(q_{o}^{2}, g_{\infty}\right)}{\left(v_{n}^{(1)}-v_{n}^{(2)}\right) B_{n}^{(2)}(g)}}
$$

and

$$
\sigma_{n}^{(2)}=\frac{\left(q_{0}^{2}\right)_{n}^{(2)}}{{ }_{0} p(g) v_{n}^{(2)}} \cdot \frac{B_{n}^{(2)}\left(g_{\infty}\right) E_{n}^{(2)}\left(q_{0}^{2}, g_{\infty}\right)-\left[v_{n}^{(1)}+A_{n}^{(1)}\left(g_{\infty}\right)\right] E_{n}^{(1)}\left(q_{0}^{2}, g_{\infty}\right)}{\left(v_{n}^{(2)}-v_{n}^{(1)}\right) B_{n}^{(2)}(o)} .
$$


This would imply that the operator

$$
B_{n}^{(2)}(\theta) L_{n}^{(j)}(p(\theta)) O_{a_{1}}^{(1)} \ldots a_{n}+\left[\frac{d}{d \phi} L_{n}^{(j)}(p(g))+A_{n}^{(j)}(g) L_{n}^{(j)}(\rho(\theta))\right] Q_{\alpha_{1}}^{(2)} \ldots a_{n}
$$

hes onomolous dimension

$$
d_{n}^{(0)}=n+2+2 v(j)
$$

for $j=1,2$. 


\section{REFERENCES AND FOOTNOTES}

1. J. D. Biorken, Phys, Rov, 179, 1547 (1969).

2. The structure functions $W_{1}$ and $w_{2}$ are defined following the convention of ref, 1 . The varioble $v=-p^{*} q / m$, whete $m$ is the nucleon mass.

3. E. D. Bloow of ol., Phys. Rev, Lett. 23, 930 (1969); M. Breidenbach of al., Phys, Rev. Lott. 23, 939 (1969).

4. Throughout this poper we spocify o four-vector $P_{\mu}$ by three spotial components $P_{1}$, $p_{2}, p_{3}$ and an imaginary time component $p_{4}=i p_{0} ; p^{2}=p_{1}{ }^{2}+p_{2}{ }^{2}+p_{3}{ }^{2}-p_{0}{ }^{2}$. We use $p=-i \gamma_{\mu} P_{\mu}$, and for Dirac spinors $\bar{U}=U^{+} \gamma_{4}$. Single porticle momentum eiganstolos $|\vec{p}\rangle$ are normalized os $\left(\vec{p} \mid \vec{p}^{\prime}\right)=2 E(2 \pi)^{3} F\left(\vec{p}-\vec{p}^{\prime}\right)$.

5. L. S. Brown, Boulder Lectures (1969). H. Loutwyter and J. Stern, Nuc. Phys. B 20, 77(1970). R. Brandt, Phys, Rev. D 1, 2808 (1970).

6. K. Wilson, Cornell report LNS-64-15(1969), Phys, Rov. 179, 1499 (1969).

7. The volidity of this oxponsion in parturbation theory has been establithed by R. Brandt, Ann. Phys. 44, 221 (1967) and W. Zimmerman in Lectures on Elementary Porticles and Qwontum Fiold Theory, MIT Pess, Combridgo, Mass. (1971).

8. Wo have omitted from Eq. (2) o third type of term containing operotors of the form $O_{\mu_{1}}^{\mu \nu} \ldots \mu_{n}$, antisymmatric under interchange of $\mu$ or $v$ with $\mu_{j}$. Such terms do not contribute to the spin averaged motrix elements under consideration.

9. Since wo will be interested in the leading light cone singularities, those operators with coefficlents whose behovior at $x^{2}=0$ is less singular then shown in Eq. (3) have been lumped into $R_{N}^{(j)}$ and $R_{N}^{(j)}$.

10. R. A. Frandt and G. Proporata, Nucl. Phys. B 27, 541 (1971); Y. Frishmon, Phys. Rov. Lett. $25,966(1970)$. The first of these roferences also gives a mathod, which wo shall 
use, of incorporating asymptotic gauge invariance inte the light cone expansion.

11. K. Symanzik, Commun, Math. Phys, 23, 49 (1971).

12. C. Callan, Phys, Rov. D 2, 1541 (1970); K. Symanzik, Commun. Math. Phys. 16, 227 $(1970)$.

13. M. Gell-Mann and F. F. Low, Phys. Rev. 95, 1300 (! I54);

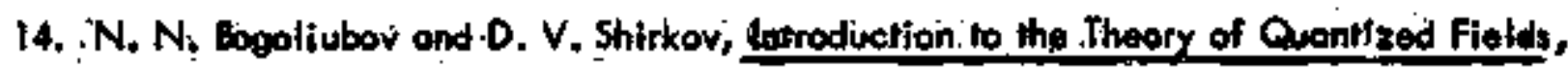
Interseience, New York (1959).

15. V. N. Gribov and L. N. Lipotov, Phys, Lett. 37 B, 78 (1971) and to be published.

16. All torms in these suns with odd n must vainish becouse of the cressing rolations $T_{2}\left(q^{2}, \omega\right)=T_{2}\left(q^{2},-w\right), T_{L}\left(q^{2}, u\right)=T_{L}\left(q^{2},-u\right)$.

17. Following Symanzik (reference 11) we shalf use the superscript AF on a function, $f\left(q^{2}, \omega\right)$, to indicate that only those terms containing the hight power of $q^{2}$ are retained in each order of perturbation theory. That is, if

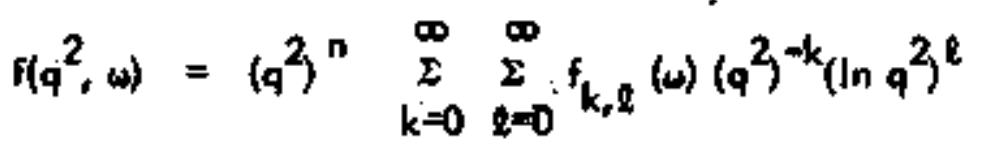

where $f_{k, j}(\omega)$ is a power sories in $g$, then $f\left(q^{2}, w\right)^{A F}$ contains only the $k=0$ Perms

$$
f\left(q^{2}, \omega\right)^{A F}=\left(q^{2}\right)^{n} \sum_{q=0}^{\infty} f_{0, p^{(w)}\left(\ln q^{2}\right)^{l}}
$$

18. C. Callan and D. Gross, Phys, Rev. Lett. 22, 156 (1969); J. Cormwoll and R. Norton, Phys. Rev. 177, 2584 (1969).

19. When appeoring on a motrix element containing the moss insertion operator $u$, the subscript A indicates that all propagators corresponding to external tines have been removed and thot all amplitudes in which $u$ acts on on external line have been dropped. 
20. Zimmerman, see roferonce 7 .

21. It should be noted that the Wilson expansion (2) is volid for matrix elements containing the moss operator $U$. In a renormalizable theory all subgraphs appearing in the matrix elements of a traceless, symmetric operator $o_{a_{1}}^{(i)} \ldots a_{n}$ of lowest canonical dimention will have degree of divergence less than or equal to zero.' Therefore, the insertion of the operater $u$ will require no additional subtractions.

22. These are the only two operators for a givan $n$ which are eharge eanjugotion aven and may hove non-vanishing, spin averaged matrix elements between two identical pacudo-sealer or fermion stotes.

23. For simplicity we use the some notation to represent analogous quantitios in the psoudo-scalar and vector theories. It should always be elear from the contextio which thory a given symbal refors.

24. S. J. Cheng and P. Fishbono, Phys, Rov. D 2 1084 (1970).

25. P. Flahbane and J. Sullivan, Fhys. Rev. D 4, 2516 (1971).

26. A. Mesoni, to be publishod:

27. The requirement (56a) is valid only if we set equal to zero the ditrect, renormalized photon coupling constont $f$, introduced in eq. (A19) and (A20).

28. If the right hond side of eq. (65a) is exponded in powers of $q^{2}$, we find $a$ term behoving as $1 / g^{2}$. This term is independent of $x^{2}$ and is therefore annihilated by the derivotives appooring in $\$$. (2).

29. For a derivation of the Callan-Symonzik equations in quantum electrodynamics that follows similar Ines, soe A. Sirlin Phys. Rov. D5, 2132 (1972).

30. Throughout our diseussion of the vector theory we work in the Feynman gatge using $\delta_{\mu v} \Delta\left(k^{2}, m, \mu\right)$ for the photon propogotor. 
31. A somowhot difforent asymptotic bohovior is implied if $g_{c}$ is a multiplo root or an essential singularity of $\beta$. For a discussion of thase various posibilities see 5. Adler, IAS preprint. 


\section{FIGURE CAPTIONS}

Flgure 1. Ladder grophs representing the "outer rainbow" amplitudes considered in the Chang-Fishbane colculation. The solid lines represent fermion propogators, the dashed lines pseudo-scaler propagotors and the wavy linus virtual photons.

Figure 2, o) Diograms representing the matrix element which determines $a_{n}^{1,1}$ to order $9^{2}$ in the peoude-scalor theory. The cross indicates insertion of the moss operator $U$ while the two photon - wwo fermion vertex represents the factor given in (C2). b) The diegreen representing the order $9^{2}$, wwo fermion matrix olement of the operator specified by eq. (B2).

Figure 3. The results of a calculotion of $o_{n}^{i, j}$ to order $g^{2}$ in the pseudo-scalar theory and these Foynmen diagroms, deseribed in Appendix C, from which their. values were obtained.

Figure 4. The quantities $o_{n}^{i, j}$ computed to order $g^{2}$ in the vector theory accompanied by the corresponding grophs $\infty$ described in Appendix C. Here the deshed lines represent vector particle propagators. 


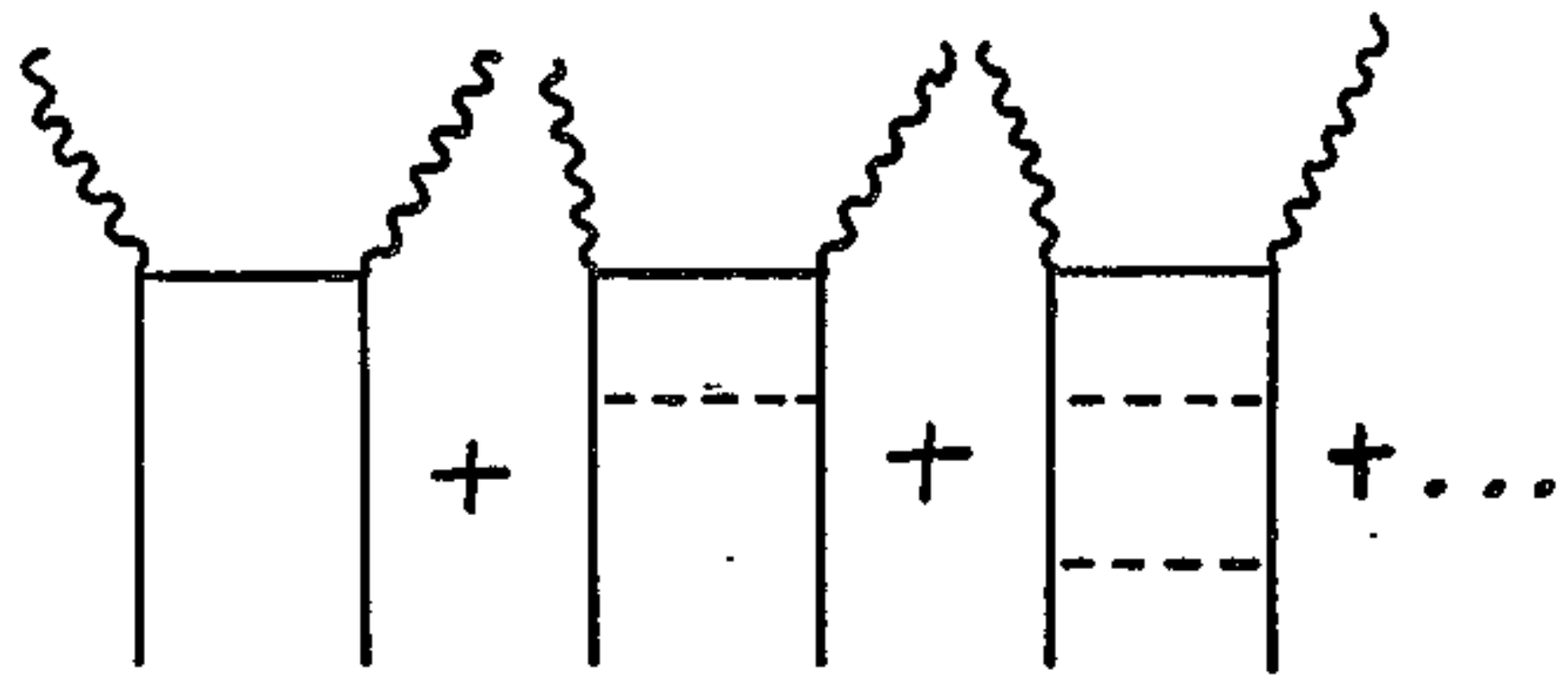

FIGURE I 
60
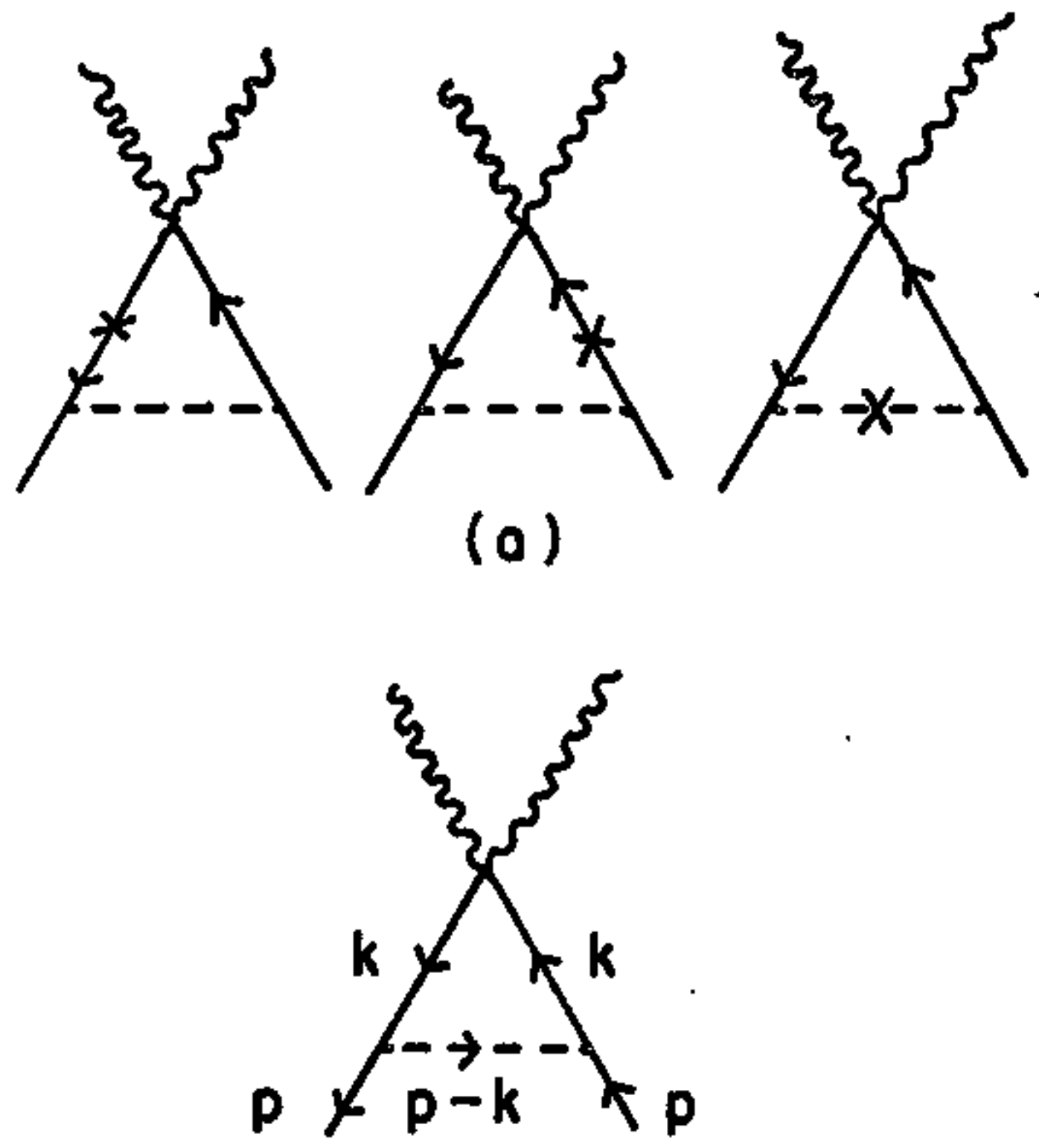

(b)

FIGURE 2 

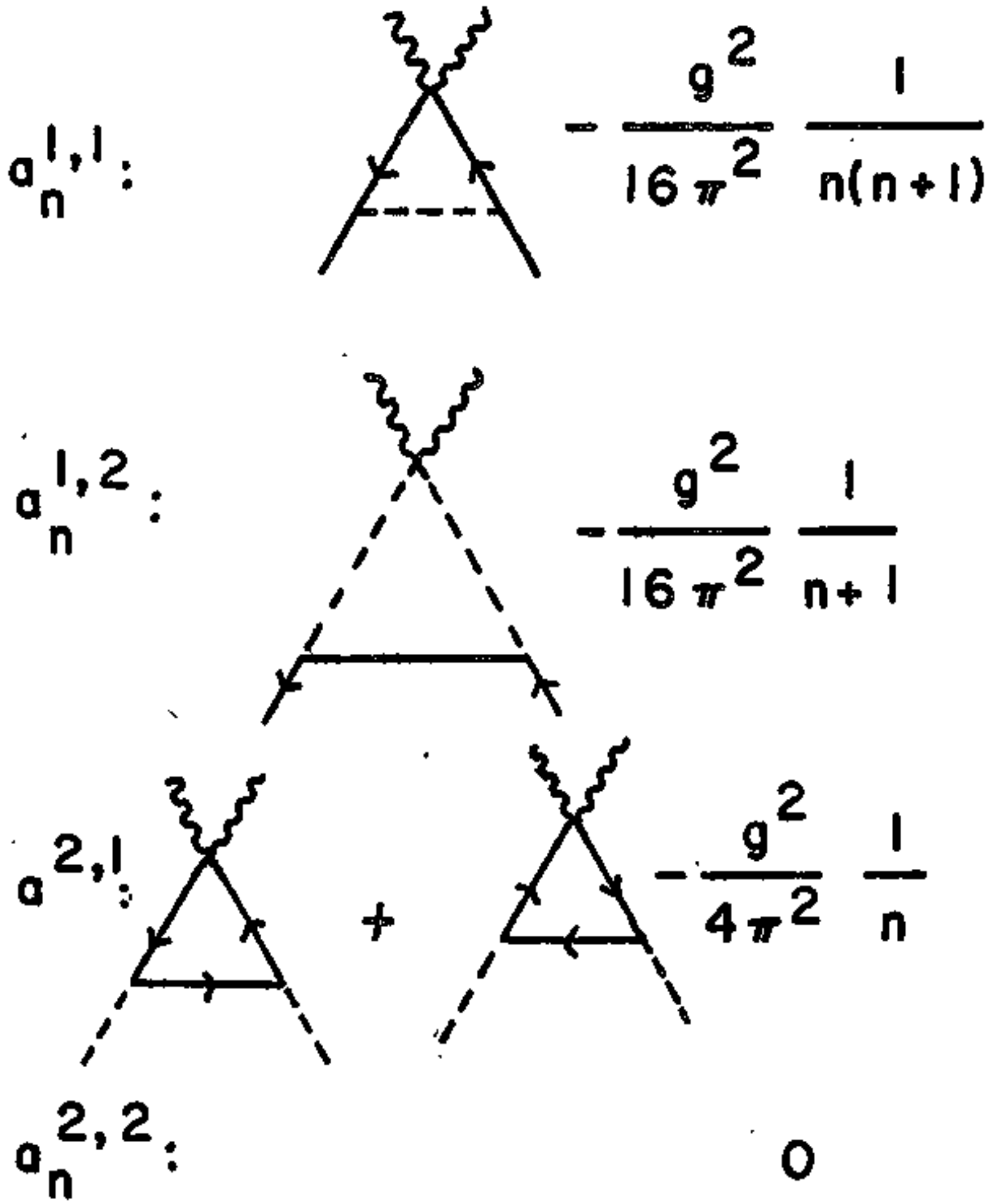

FIGURE 3 


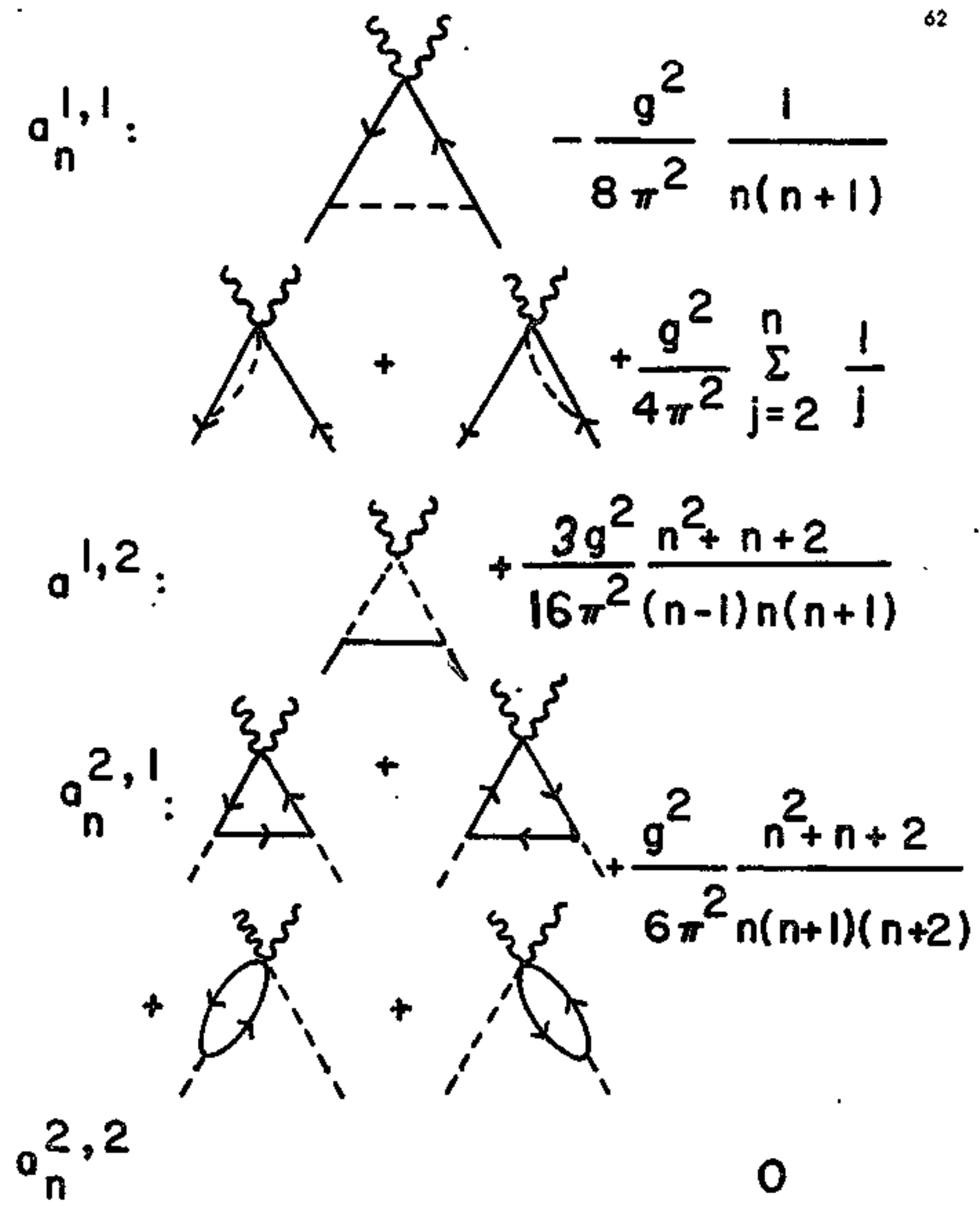

FIGURE 4 